\title{
MHD simulations of quadrupolar paleomagnetospheres
}

\author{
J. Vogt and B. Zieger ${ }^{1}$ \\ School of Engineering and Science, International University, Bremen, Germany
}

A. Stadelmann and K.-H. Glassmeier

Institut für Geophysik und extraterrestrische Physik, Technische Universität, Braunschweig, Germany

T. I. Gombosi, K. C. Hansen, and A. J. Ridley

University of Michigan, Ann Arbor, Michigan, USA

Received 2 October 2003; revised 29 July 2004; accepted 25 October 2004; published 24 December 2004.

[1] During geomagnetic polarity transitions the Earth's internal magnetic field is expected to deviate significantly from the dipolar configuration observed today, and higher-order multipoles can become important. Their effects on the magnetosphere are investigated in this study by means of MHD simulations. We look at quadrupolar configurations and vary the parameters that are expected to be most important in determining the largescale current systems and the field line topology of such paleomagnetospheres, namely, (1) the orientation of the internal field as well as (2) the orientation of the interplanetary magnetic field (IMF) with respect to the solar wind flow, and (3) the quadrupole shape parameter which controls the topology of the internal field. Zero IMF conditions yield reference cases which allow to locate the magnetopause that otherwise can be obscured by field line merging. Magnetopause currents and cross-tail currents in quadrupolar configurations are contrasted with the tail current system in the present-day dipolar magnetosphere. Interplanetary magnetic fields with a nonzero component perpendicular to the solar wind flow are found to have a much stronger effect on the global magnetospheric configuration than a purely parallel or antiparallel IMF. Since a quadrupole field is more structured than a dipole field, conditions that favor field line merging at the dayside are met regularly in quadrupolar magnetospheres. Solar wind-magnetosphere coupling triggered by reconnection should thus be persistent rather than strongly controlled by solar wind parameters. The investigation presented in this report is part of a multi-institutional collaboration to model the effects of geomagnetic polarity transitions on the Earth's environment in space and the upper and middle atmosphere. INDEX TERMS: 2740 Magnetospheric Physics: Magnetospheric configuration and dynamics; 2784 Magnetospheric Physics: Solar wind/magnetosphere interactions; 1503 Geomagnetism and Paleomagnetism: Archeomagnetism; KEYWORDS: paleomagnetosphere, MHD simulation, geomagnetic polarity reversal, quadrupole magnetosphere, magnetospheric current system, magnetic field topology

Citation: Vogt, J., B. Zieger, A. Stadelmann, K.-H. Glassmeier, T. I. Gombosi, K. C. Hansen, and A. J. Ridley (2004), MHD simulations of quadrupolar paleomagnetospheres, J. Geophys. Res., 109, A12221, doi:10.1029/2003JA010273.

\section{Introduction}

[2] The Earth's magnetosphere results from the interaction of the solar wind with the geomagnetic field generated in the planetary interior. It has become textbook knowledge [e.g., Baumjohann and Treumann, 1997] that magnetospheric activity and dynamics are controlled by variations of solar wind parameters like the dynamic pressure and the interplanetary magnetic field (IMF). In particular, magnetic

\footnotetext{
${ }^{1}$ Also at Geodetic and Geophysical Research Institute, Hungarian Academy of Sciences, Sopron, Hungary.

Copyright 2004 by the American Geophysical Union. 0148-0227/04/2003JA010273
}

substorms can be understood as global reconfigurations of the magnetospheric topology from closed to open type [Dungey, 1961] triggered by a change in IMF orientation. Of key importance in this context is the concept of reconnection or field line merging. For a recent review on reconnection, see Haerendel [2001] or Priest and Forbes [2000].

[3] If compared to effects of solar wind variability, temporal changes of the internal geomagnetic field can be safely disregarded on timescales of the order of decades. On archeological timescales, however, the Earth's dipole moment can vary by a factor of two [Merrill and McElhinny, 1983; Jacobs, 1994]. The currently observed decrease of the dipole field strength of about $1 \%$ in 20 years is also indicative of such a variability. The effects of a changing 
dipole moment on various magnetospheric regions like the magnetopause, the plasmasphere, and the ring current region were studied by Siscoe and Chen [1975] who coined the term "paleomagnetosphere." These aspects were further developed in a comparative study of planetary magnetospheres [Siscoe, 1979]. Vogt and Glassmeier [2001] addressed the consequences for large-scale current systems to provide scaling relations for quantitative magnetospheric models.

[4] On geological timescales, even stronger geomagnetic variations take place. The most dramatic changes of the internal geomagnetic field are called polarity transitions or field reversals because then the Earth's dipole axis changes its orientation by about $180^{\circ}$. Paleomagnetic measurements indicate that the duration of a polarity transition event is of the order of several thousand years. The topology of the transition field is still a matter of debate among paleomagnetists [Merrill and McFadden, 1999]. A convenient means for putting (local) paleomagnetic records taken at different sites into a global context is the VGP (virtual geomagnetic pole) representation: a single magnetization direction measurement allows to uniquely construct a centered dipole axis that would lead to this particular measurement at the observation site, and the VGP is defined as the intersection point of the constructed dipole axis with the Earth's surface [Merrill and McElhinny, 1983]. Accordingly, a magnetization profile can be associated with a whole sequence of centered dipole axes and a path of VGPs. If a reversal was recorded, this procedure yields a VGP path from one hemisphere to the other, which suggests that the dipole axis should reside around the geographical equator for some period of time, and this is indeed a conceivable transition field configuration. In the paleomagnetospheric context this scenario was addressed by Saito et al. [1978], who showed that strong diurnal variations can be expected even for quiet solar wind conditions because of the Earth's rotation. This type of paleomagnetosphere is investigated in detail by means of MHD simulations in a companion paper [Zieger et al., 2004].

[5] Although paleomagnetic records can be uniquely represented in terms of VGP paths, there is no compelling evidence for interpreting the data in terms of pure dipole fields. Many different geomagnetic field configurations can lead to the same local magnetization sequence. In particular, higher-order multipoles have been successfully utilized in the interpretation of paleomagnetic records [e.g., Williams and Fuller, 1981; Clement and Kent, 1985; Clement, 1991]. Furthermore, large-scale geodynamo simulations [e.g., Glatzmaier and Roberts, 1996] also indicate that higherorder multipoles should significantly contribute to the Earth's core field during polarity transitions. The resulting magnetospheric structure has not yet received very much attention. Siscoe and Crooker [1976] looked at the location of the auroral zones in a quadrupolar magnetosphere. Rishbeth [1985] discussed principal features that might exist in a quadrupolar paleoionosphere. Vogt and Glassmeier [2000] studied the location of trapped particle populations in a quadrupolar paleomagnetosphere. The few quantitative models of quadrupolar magnetospheres that exist are either two-dimensional [Leubner and Zollner, 1985; Biernat et al., 1985; Starchenko and Shcherbakov, 1991] or highly (spherically) symmetric [Willis et al., 2000]. A quantitative three-dimensional model that treats the dipolar and the quadrupolar as well as the mixed case in a more realistic geometry has been developed at the TU Braunschweig [Stadelmann, 2004].

[6] This report is supposed to investigate the electrical current systems and the magnetic field line topologies of quadrupolar magnetospheres by means of MHD simulations. We proceed as follows. First, we explain the chosen methodology (section 2) and which parameters are varied in this study, namely, the IMF orientation and the topology parameter of the internal geomagnetic quadrupole field as well as its orientation. In section 3 we deal with axisymmetric quadrupoles. Unlike dipolar core fields, quadrupoles may exhibit neutral lines. Their effects are investigated in section 4 . We conclude with a brief summary in section 5 where this project is put into a larger context.

\section{Methodology}

[7] Magnetosphere formation depends on a number of variables which can be loosely grouped into (1) the set of solar wind parameters, (2) the coefficients of the internal planetary magnetic field, and also (3) the set of atmospheric/ ionospheric parameters. Since in this study we focus on current systems and magnetic field line topology in the outer magnetosphere, ionospheric parameters are only implicitly considered by imposing conditions at the inner boundary of the simulation domain, i.e., the interface to the planetary atmosphere, which mimic the response of a conductive ionosphere (for details, see Appendix B). We thus make no attempt to analyze or interpret electrical currents close to the planetary surface. The parameter space spanned by solar wind variables and geomagnetic field coefficients, however, still remains highly dimensional. Within the scope of this paper we can afford to vary a few key control variables which are described in the following paragraphs.

\subsection{Solar Wind Parameters}

[8] In order to identify the parameters that mainly control the topologies of quadrupolar magnetospheres, it is instructive to look at the dipole case first. The size of such a magnetosphere is mainly controlled by a single hydrodynamic solar wind parameter, namely, the kinetic energy density or ram pressure [Martyn, 1951; Siscoe and Chen, 1975; Vogt and Glassmeier, 2001] because it is considerably larger than the magnetic energy density of the IMF and the thermal energy density. On the other hand, magnetospheric topology is governed by the orientation of the IMF. As already proposed by Dungey [1961], one distinguishes between "open" type magnetospheres, where a significant amount of solar magnetic flux and energy can enter the geomagnetic cavity, and a "closed" type, with much less solar wind-magnetosphere coupling. These concepts are now widely accepted in the interpretation of magnetic substorms and have entered space physics textbooks [e.g., Baumjohann and Treumann, 1997]. Since in this study we intend to focus on the topologies of quadrupolar magnetospheres, we keep hydrodynamic solar wind parameters like the ram pressure fixed. Only the orientation of the IMF is varied. Zero field conditions are chosen as a reference case, and then the effects of different orientations with respect to 


$$
\eta=0.0
$$
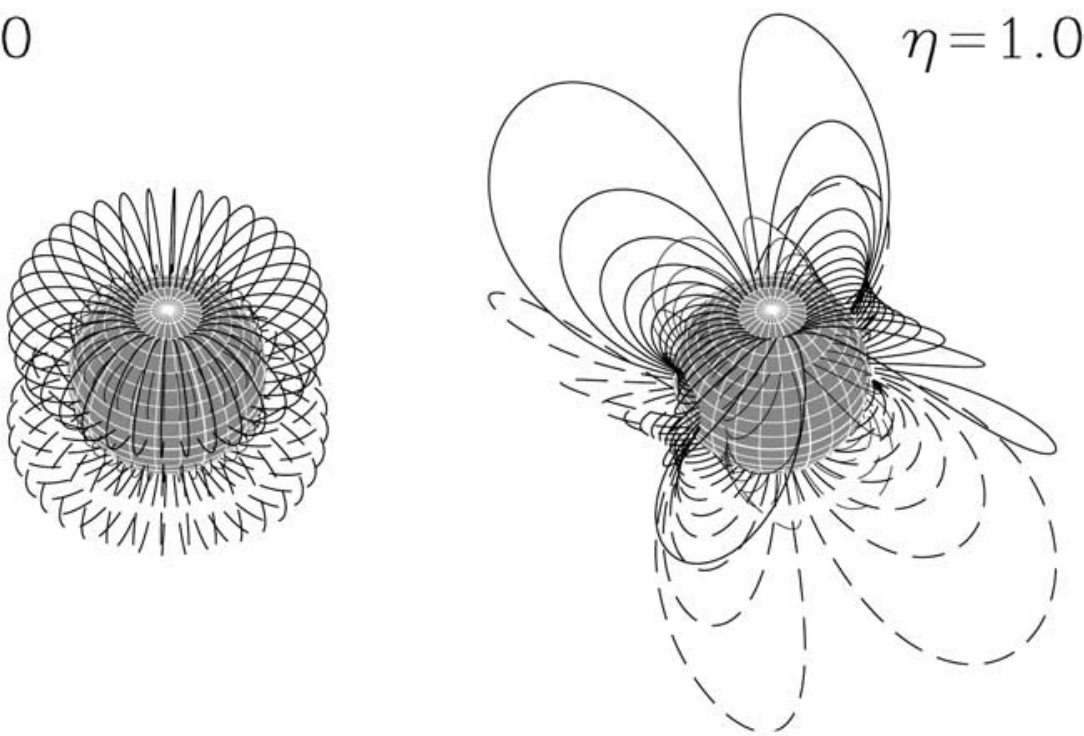

Figure 1. The two classes of quadrupole fields used in this study. (left) The shape parameter $\eta=0$ corresponds to an axisymmetric quadrupole. (right) The case $\eta=1$ yields a quadrupole with four poles, and a neutral line is located perpendicular to both polar axes.

the solar wind flow direction are studied: parallel, antiparallel, perpendicular, and oblique.

[9] Such a reference case is very useful for magnetopause identification. By definition, the magnetopause separates planetary magnetic field lines from the IMF. This concept is strictly applicable only if planetary and solar wind magnetic field lines do not connect, i.e., in closed magnetospheres. In the presence of field line merging which yields open magnetospheres, the magnetopause is no longer unambiguously defined. Hence for a given internal quadrupole field configuration, we first identify the magnetopause in the zero IMF case with the location of the last planetary magnetic field line and find the associated Chapman-Ferraro currents. This current system persists structurally for nonzero IMF even if some interplanetary magnetic lines merge with terrestrial ones.

\subsection{Geomagnetic Field}

[10] The internal geomagnetic field parameters form the second set of variables which control magnetosphere formation. The size of a dipolar magnetosphere depends on the (scalar) dipole moment whereas magnetospheric topology changes with the dipole axis orientation [e.g., Zieger et al., 2004]. Therefore with an axisymmetric quadrupole as the core field, we expect the size of the magnetosphere to be controlled by the magnitude of the quadrupole moment. The topology should change with the orientation of the quadrupole symmetry axis. We consider orientations parallel and perpendicular to the solar wind flow.

[11] A general quadrupole field can be described using five parameters, e.g., the five coefficients of a spherical harmonics expansion or the five independent components of a quadrupole tensor in cartesian representation (note that quadrupole tensors are symmetric and have zero trace). Through tensor diagonalization it can be shown [Vogt and Glassmeier, 2000] that three out of these five degrees of freedom control the orientation of a general quadrupole field, one determines the absolute field strength, and the remaining one can be interpreted as a quadrupole topology or "shape" parameter $\eta$ in the range $[-1,1]$ (for details, see also Appendix A). The axisymmetric case is given by $\eta=0$. The extreme cases $\eta= \pm 1$ yield quadrupoles with neutral lines which are addressed in section 4 . The field lines for the axisymmetric configuration $\eta=0$ and the neutral line case $\eta=1$ are sketched in Figure 1. For details about the topological distinction between quadrupolar fields and implications for drift orbits of trapped particles the reader is referred to Vogt and Glassmeier [2000].

\subsection{Model}

[12] The MHD simulation code BATS-R-US used in this study was developed at the University of Michigan. It solves the ideal MHD equations on a self-adaptive grid to yield high resolution at magnetospheric boundaries. Since this study aims at a topological classification of magnetic field lines and current systems of quadrupolar magnetospheres in terms of the parameters given above, we do not model the full magnetospheric dynamics but present solutions of steady-state runs. Note that it is not clear if magnetospheres in general support strictly stationary solutions [Erickson and Wolf, 1980]. Adiabatic convection from the tail to the inner magnetosphere has been argued to yield such large pressures that the term "pressure balance inconsistency" was coined and suggested as a key element in substorm evolution. This question clearly requires some more attention: it not only affects steady-state simulations (which are frequently used to describe magnetospheric phenomena) but also basically all quantitative magnetospheric models because they typically disregard the time dimension. Within the context of this work, however, steady-state solutions of MHD simulations (possibly supported by some unavoidable numerical dissipation, see below) are expected to produce at least a set of representative average magnetospheric configurations which allow to aim at a first topological classification of quadrupolar magnetospheres. 
[13] In accordance with the supersonic and superalfvénic solar wind, the outer boundary conditions in the simulation box were set to inflow or outflow, and the inner boundary condition (at the planet) used here corresponds to a highly conducting ionosphere. In each case the magnitude of the planetary quadrupole was chosen such that the resulting maximum surface field strength was $10 \%$ of the present-day value. The basic features of the BATS-R-US code including the set of underlying equations and the solution strategy are briefly summarized in Appendix B. A detailed description can be found in the work of Powell et al. [1999].

[14] Magnetic reconnection is a phenomenon that requires some kind of nonideal behavior which is not present at the partial differential equation (PDE) level of the underlying set of equations. However, it is well known that discretization in general introduces dissipation and dispersion into the system. Various factors like grid resolution or the type of PDE solver influence the amount of numerical dissipation present in the simulation code, and valid aspects of a solution must be robust with regard to these numerical factors. The problem studied here turned out to be rather challenging and required some effort to optimize the numerical grid and to go to the limit of available computer resources in terms of memory. The results that will be discussed in the following were obtained on a numerical grid of more than two millions cells and a resolution of $0.125 R_{E}$ in key magnetospheric regions like the dayside magnetopause current sheet and bow shock. Increasing the resolution might still influence the thickness or the extent of current sheets. However, the structure and the locations of the current sheets as well as the magnetic field line topology were essentially unaffected when the total number of cells was varied by a factor of two and when different PDE solvers or grid refinement criteria were used. The solution aspects addressed in this study are thus expected to be robust with regard to the grid resolution and other purely numerical factors.

[15] We may add that the most popular global magnetospheric MHD codes, including the BATS-R-US code used in this study, have been compared against each other and also reasonably well validated against a variety of spacecraft and ground-based measurements [e.g., Janhunen et al., 1995; Lyon et al., 1998; Fedder et al., 1998; Raeder et al., 1998; Winglee and Menietti, 1998; Gombosi et al., 1998; Raeder et al., 2001; Slinker et al., 2001; White et al., 2001; Siscoe et al., 2002; Kabin et al., 2003; Rae et al., 2004; Ohtani and Raeder, 2004; Nakata et al., 2004].

\subsection{Graphical Representation}

[16] We use two types of diagrams in the following sections.

[17] 1. Magnetic field lines in selected cross sections of the three-dimensional simulation domain are shown on the diagrams of the first type (Figures 2, 4, 5, and 7). Background colors (filled contour representation) indicate regions where electrical currents flow perpendicular to these planes: currents into the plane are indicated by red/magenta, currents out of the plane are indicated by yellow/green, and current values close to zero are indicated by cyan. (In the grayscale versions of these figures, red/magenta is replaced by white, yellow/green is replaced by dark gray, and cyan is replaced by light gray.) Note that the magnetic field lines shown in the diagrams are only meant to visualize the magnetic topology; therefore the density of field lines is not indicative of the magnetic field strength.

[18] 2. Current vectors projected onto the plane $x=-4 R_{\mathrm{E}}$ (perpendicular to the solar wind flow direction, magnetotail region) are displayed on the diagrams of the second type (Figures 3 and 6). To visualize the regions of current flow better, the magnitudes of projected current vectors are drawn using a logarithmic color scale.

\section{Axisymmetric Quadrupoles}

[19] All simulations in this section were carried out with an axisymmetric quadrupole as the internal magnetic field, i.e., the quadrupole shape parameter $\eta=0$. The orientation of this field with respect to the solar wind can be conveniently characterized through the angle between the symmetry axis and the solar wind flow direction. We discuss first the pole-on configuration where the symmetry axis is parallel to the solar wind flow. In a second subsection the equator-on configuration is addressed, here the symmetry axis is perpendicular to the solar wind flow. The solutions for both cases and different IMF conditions are displayed in Figures 2 and 3. As mentioned above, zero IMF conditions (upper panels in both figures) are supposed to establish reference solutions which allow to identify the magnetopause and the associated Chapman-Ferraro current system.

\subsection{Symmetry Axis Parallel to the Solar Wind Flow}

[20] The panels on the left of Figure 2 show simulation results for the axisymmetric pole-on case but different IMF conditions. The upper three solutions are axisymmetric because the IMF component perpendicular to the solar wind flow direction is zero. Fully axisymmetric solutions can no longer be expected if the perpendicular IMF component is nonzero.

\subsubsection{Zero Perpendicular IMF}

[21] In the zero IMF case (upper left panel of Figure 2) the majority of magnetic field lines that emerge from the planetary surface connect back to the Earth. Only in a small region around the dayside pole the magnetic field lines are draped around the whole magnetosphere, and also around the nightside pole the emerging field lines extend far into the magnetotail. At the nose of the magnetopause a magnetic singular point similar to the cusp in dipolar magnetospheres has formed. The magnetopause is clearly visible as the layer of Chapman-Ferraro currents around the outermost magnetic field line. These magnetopause currents are most pronounced on the dayside but loose intensity in the tail region so that there they are no longer visible in this graphical representation. Further inward, a second sheet current shows up at about $x=1 R_{\mathrm{E}}$ and extends far into the magnetotail. For brevity, we refer to this sheet current as the "outer tail current." It is located between the magnetopause and a region of closed magnetic field lines that emerge from the nightside quadrupolar hemisphere. Just inside this set of field lines, a third sheet current appears in the magnetotail which is less pronounced than the outer tail current but clearly visible. This current may be termed the "inner tail current." Note that the axial symmetry of the problem implies that all currents flow in circles around the symmetry axis. 

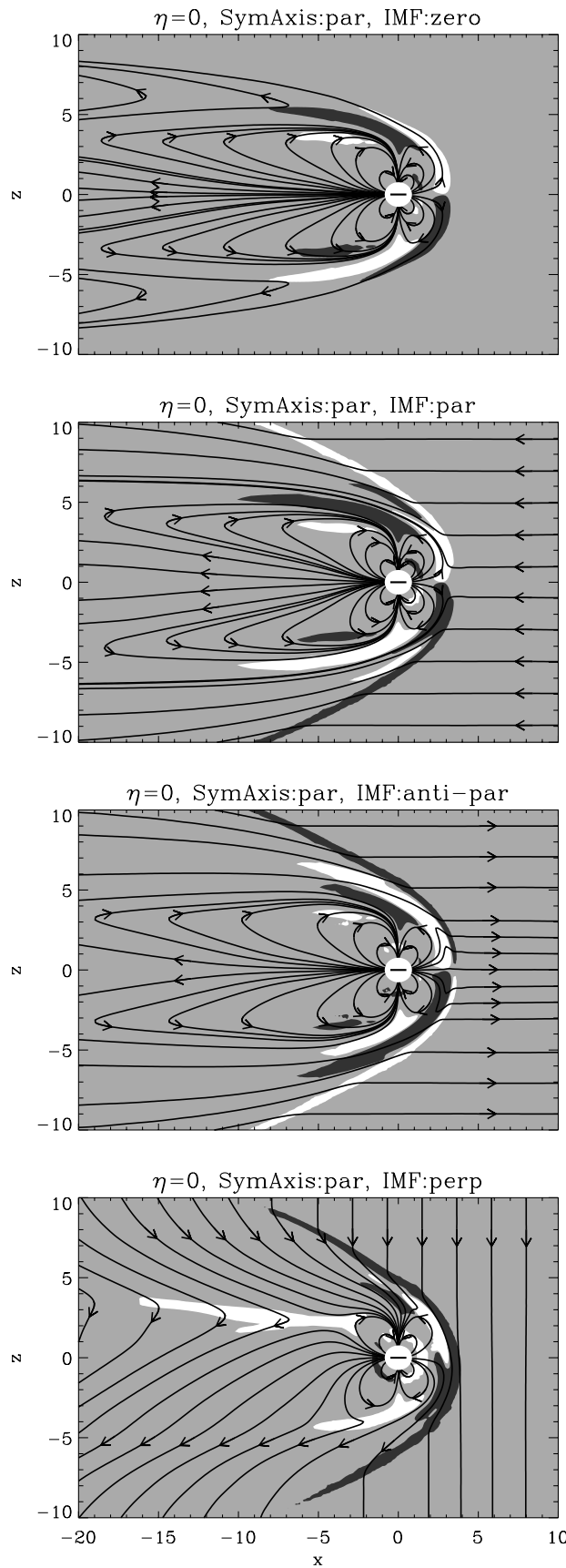

$\eta=0$, SymAxis:perp, IMF:zero

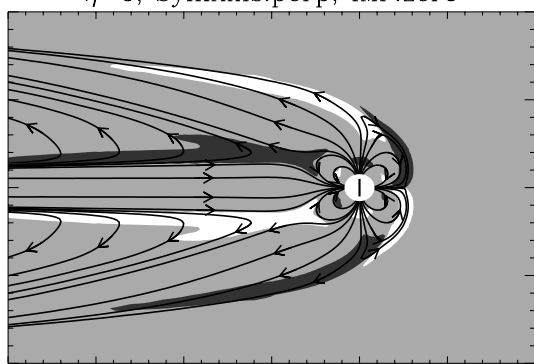

$\eta=0$, SymAxis:perp, IMF:par

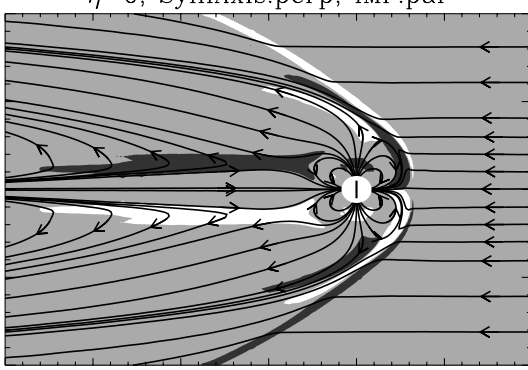

$\eta=0$, SymAxis:perp, IMF:anti-par

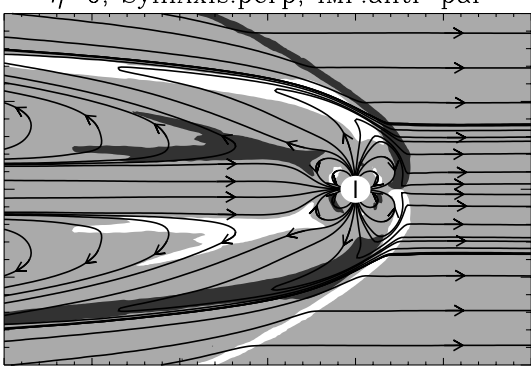

$\eta=0$, SymAxis:perp, IMF:perp

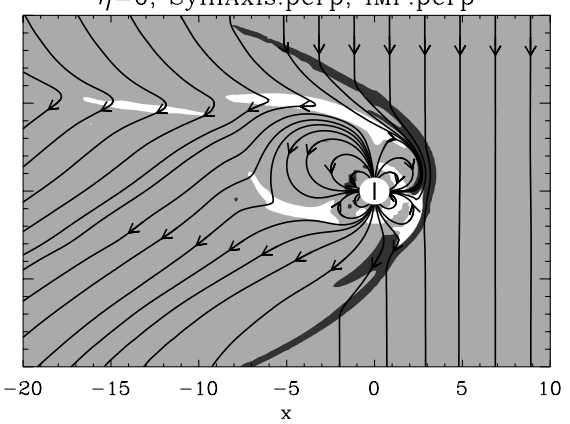

Figure 2. Axisymmetric quadrupolar core fields: magnetic field lines (solid) and $y$ component of current density (filled contours) in the plane $y=0$. See color version of this figure at back of this issue.

[22] The upper left panel of Figure 3 shows the current vectors projected onto the plane $x=-4 R_{\mathrm{E}}$. The diagram confirms that the magnetopause current (outermost current vectors, clockwise rotation), the outer tail current (anticlockwise orientation of current vectors), and the inner tail current (innermost current vectors, clockwise rotation) are indeed three separate current systems.

[23] The second panel on the left-hand side in Figure 2 shows that the magnetospheric field line topology does not change much with respect to the zero IMF case if the solar wind carries an embedded magnetic field oriented parallel to the flow direction. Basically, all terrestrial field lines still connect back to the Earth, field lines close to the dayside pole are draped around the magnetosphere, and those close to the nightside pole are stretched into the far tail. Interplanetary magnetic field lines follow the solar wind flow around the magnetosphere and do not connect to the Earth. This means that this configuration can be considered as a closed-type magnetosphere. The dayside singular cusp-like point is still there because the solar wind field and the terrestrial field are oppositely directed close to this point and do not interconnect.

[24] The magnetospheric current systems found in the zero IMF case are modified in intensity but remain structurally unchanged. Two additional current systems can be seen outside the magnetopause that both are associated with 

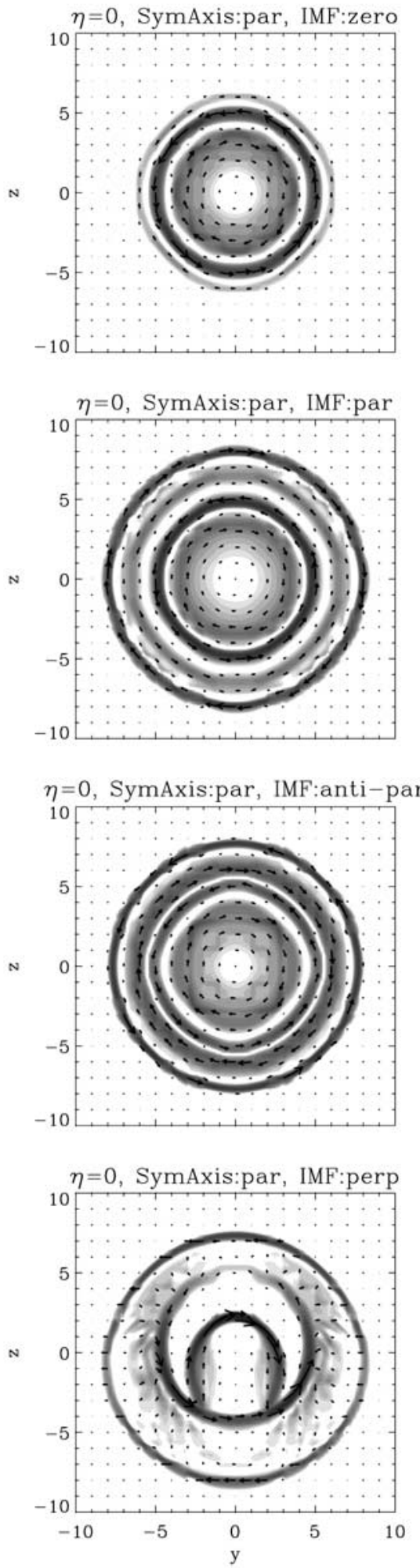
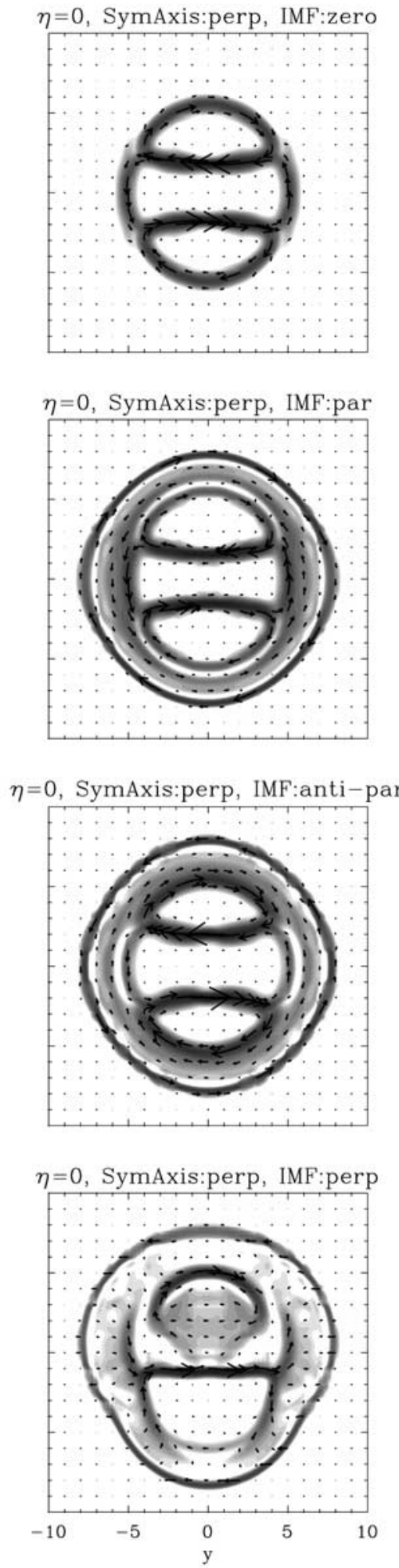

Figure 3. Axisymmetric quadrupolar core fields: current vectors projected onto the plane $x=-4 R_{\mathrm{E}}$. See color version of this figure at back of this issue.

the distortion of interplanetary magnetic field lines as the solar wind flows around the magnetosphere. The outermost current system is caused by the kink in magnetic field at the bow shock which suggests the term bow shock current.
Inside of the bow shock, the magnetic field tends to bend backward to its asymptotic parallel direction. This yields a current system that flows opposite to the bow shock current, and it may be termed the magnetosheath current. The two 
nonmagnetospheric current systems are visible in second left-hand panel of Figure 3 as the two outermost current regions. As before in the zero IMF case, the two innermost current systems are the inner tail current and the outer tail current. The magnetopause current has a sense of rotation opposite to the magnetosheath current but is too weak to be resolved in this figure.

[25] If the IMF is antiparallel to the solar wind flow (see the third panel on the left in Figure 2), the configuration favors field line merging in a small region around the dayside pole which implies that the singular cusp-like point has vanished. However, the magnetospheric configuration as whole remains again essentially unchanged. If compared to the parallel IMF case, the bow shock current and the magnetosheath current now have the opposite sense of rotation. This implies that the magnetopause current merges with the magnetosheath current and forms one thick current layer. This can also be seen in the third panel on the lefthand side of Figure 3.

[26] Note that in these simulations the poles on the symmetry axis of the internal quadrupole were divergent (whereas the geomagnetic equatorial plane located at $x=0$ was a field line convergence region). Since the field line topology only depends on the relative orientation of the IMF and the internal field, the convergent pole and antiparallel IMF case yields the same field line pattern as the divergent pole and parallel IMF case. The same symmetry argument implies that the field line topology of the convergent pole and parallel IMF case is identical with the topology of the divergent pole and antiparallel IMF case.

[27] The three IMF orientations discussed so far have led to fully axisymmetric solutions. This is no longer the case if the IMF has a nonzero component perpendicular to the SunEarth line. It should also be noted that the magnetosheath current is a special current systems associated with purely parallel or purely antiparallel IMF. If the solar wind magnetic field has perpendicular orientation, it is no longer visible.

\subsubsection{Nonzero Perpendicular IMF}

[28] If the IMF is perpendicular to the solar wind flow direction, then there is always one hemisphere of a pole-on axisymmetric quadrupole where the terrestrial field lines are antiparallel to the IMF. This geometry yields dayside reconnection as shown in the lower left panel of Figure 2. Magnetic flux is transported along the magnetopause in the upper part of this figure, and reconnection takes place also in the tail region. The configuration in this hemisphere thus compares to an open-type dipolar magnetosphere. As the interplanetary magnetic field lines in the lower hemisphere of the figure drape around the nose of the magnetopause, they find antiparallel counterparts at the flanks of the magnetosphere which also gives rise to field line merging. A nonzero perpendicular IMF component thus has a much stronger effect on the magnetospheric field line topolgy than a purely parallel or antiparallel IMF.

[29] The Chapman-Ferraro currents on the dayside magnetopause still flow mainly around the Sun-Earth axis. On the nightside, the magnetopause currents are too weak to be resolved. The circular tail current systems in the inner magnetosphere have transformed into a more complex and essentially three-dimensional current configuration. Although projected current vectors offer only incomplete information about the current flow lines in such a case,

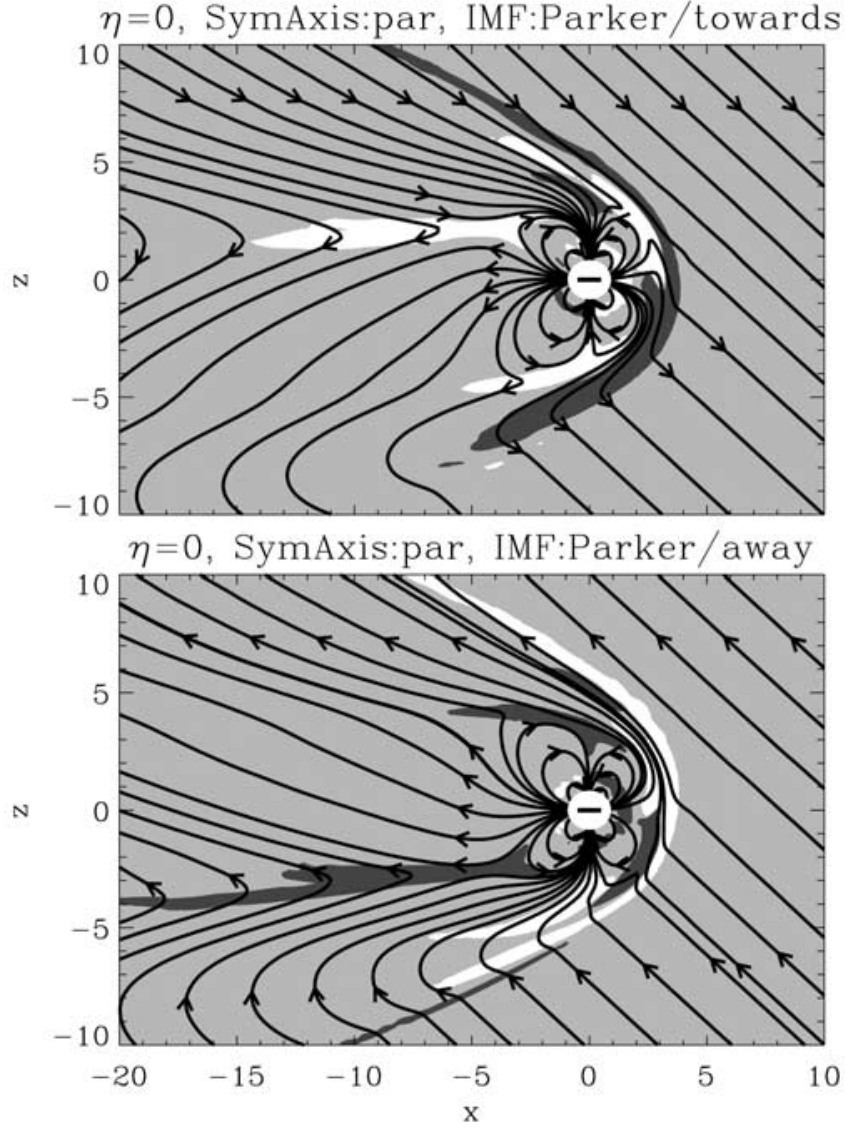

Figure 4. Axisymmetric quadrupolar core fields, oblique IMF: magnetic field lines (solid) and $y$ component of current density (filled contours) in the plane $y=0$. See color version of this figure at back of this issue.

the lower left panel of Figure 3 looks as if due to the solar wind-magnetosphere interaction associated with the field line merging sites, the inner tail current has merged at its bottom end with the outer tail current.

[30] If the IMF is neither fully perpendicular nor parallel or antiparallel to the solar wind flow direction but oblique, one might expect the resulting magnetospheric configuration to be a mixture of the (anti-)parallel and the perpendicular IMF cases. The two panels of Figure 4 show configurations where the angle between the solar wind flow direction and the IMF is roughly $45^{\circ}$ as for the Parker spiral. The field topology shown in the upper panel (toward sector) is very similar to the perpendicular IMF case shown in the lower left panel of Figure 2, and also the large-scale current systems are in almost the same places. Analogous conclusions can be drawn from the lower panel of Figure 4 that shows the magnetospheric configuration if the IMF corresponds to a Parker spiral in the away sector. Hence in this context the parallel IMF component appears to be a less import control parameter than the perpendicular IMF component.

\subsection{Symmetry Axis Perpendicular to the Solar Wind Flow}

[31] Now we turn our attention to a different category of quadrupolar magnetospheres, namely, those where the symmetry axis of an axisymmetric internal field is oriented 
perpendicular to the solar wind flow. Here the Sun-Earth line is parallel to the geomagnetic equator, so we refer to this configuration briefly as the "equator-on case." Although at first glance the geometry seems similar to the present-day dipolar magnetosphere, there are essential differences in the topologies because in the geomagnetic equatorial region the quadrupolar field lines are radial instead of tangential. Note that this internal field geometry does not yield axisymmetric solutions.

[32] The zero IMF case shown in the upper right panel of Figure 2 yields a configuration which is symmetric with respect to the geomagnetic equatorial plane. One finds closed field lines in the inner magnetosphere. Open field lines emerge from the polar caps and extend far into the magnetotail. The outermost such field line marks the boundary of the magnetosphere in the tail region. The dayside magnetopause is marked by the field lines that emerge in the region around the dayside geomagnetic equator and connect to one of the two poles. In both the top and the bottom hemisphere we thus find a configuration which is topologically similar to the present-day dipolar case. In particular, Chapman-Ferraro currents also form vortices around the cusps. However, unlike the current flow in the dipolar case, quadrupolar magnetopause currents change direction at the geomagnetic equatorial plane. One may also note that besides the two cusps, the geomagnetic equator constitutes another magnetic singularity region which is not merely a point but an extended line.

[33] The behavior of dayside magnetopause currents suggests that we should find analogous similarities and differences in the tail region where the cross-tail current is a major characteristic in a dipolar magnetosphere. The upper right panels of Figures 2 and 3 confirm this conjecture. Two cross-tail currents show up in the quadrupolar magnetosphere, one in each hemisphere, which both connect to the magnetopause current system.

[34] The second panels on the right-hand sides of Figures 2 and 3 show that the situation does not change much if a parallel IMF component comes into play. In a small region around the subsolar point on the magnetopause, terrestrial field lines are connected to the IMF. The tail magnetopause current is less pronounced than in the zero IMF case because interplanetary magnetic field lines are parallel to terrestrial ones emerging from the polar regions which reduces magnetic shear at the flanks of the magnetosphere. Since the tail magnetopause currents close inside the magnetosphere through the systems of cross-tail currents, these current systems are also reduced in intensity. The bow shock current and the magnetosheath current do not change this picture, they are simply superposed to the magnetospheric current systems and do not connect to them.

[35] The field line topology is altered if the IMF is directed antiparallel to the solar wind flow direction. The third panel on the right-hand side of Figure 2 shows how the polar field lines are stretched far out into the magnetotail and then connect back to the IMF at the dayside. The configuration can be interpreted as the result of field line merging initiated at the nose of the magnetopause, transport of magnetic flux over the polar caps, and merging in the nightside magnetosphere, which suggests that this can be considered to be an open-type magnetosphere. The whole process is supported by the magnetic field geometry at the magnetospheric flanks where interplanetary magnetic field lines find antiparallel couterparts of planetary origin. The flank geometry also suggests that the tail magnetopause currents and thus also the cross-tail currents should be more intense which indeed is the case, see also the third panel on the right-hand side of Figure 3. As in the parallel IMF case, the bow shock current and the magnetosheath current simply flow around the magnetospheric current systems and do not interfere with them.

[36] As already noted before, the topological effects of parallel and antiparallel IMF depend on the whether the poles on the symmetry axis are convergent or divergent, and the same statement as above can be made here.

[37] In contrast to the three IMF conditions discussed so far for the axisymmetric equator-on quadrupole case, the configuration for perpendicular IMF conditions (lower right panel in Figure 2) is by no means symmetric with respect to the geomagnetic equatorial plane. The hemisphere in the lower part of the figure shows two reconnection sites, one on the dayside and the other one in the tail, just as in a dipolar open-type magnetosphere. Apart from the reconnection site in the cusp region in the upper part of the figure, the upper hemisphere resembles more a closed-type dipolar magnetosphere.

[38] The loss of symmetry between the two hemispheres makes it difficult to infer the connectivity of electrical current systems. The system of tail magnetopause currents and cross-tail currents are converted into a three-dimensional current configuration which cannot be unambiguously inferred from the lower right panel of Figure 3. From the tail current in the upper hemisphere, only the magnetopause contribution seems to have survived in intensity, the crosstail current is at least distributed over a larger region than in the zero IMF case. The cross-tail current in the lower part of the figure has basically maintained its strength and its structure but the magnetopause currents which feed this sheet current have changed shape and are now essentially threedimensional and difficult to track.

\section{Quadrupoles Exhibiting Neutral Lines}

[39] On a spherical surface around a central dipole the field strength varies by a factor of 2 but not more. The situation changes significantly if higher-order multipoles are considered. Regions of very weak field strength can appear in such configurations, and even neutral lines (also called null lines) may show up, e.g., in the quadrupole $\eta=1$ case. In this section we look at the magnetospheric configurations that result from a central $\eta=1$ quadrupole. We investigate how the geometry changes with different IMF conditions and different angles between the neutral line and the solar wind flow direction.

[40] It should be stressed that the two-dimensional displays of field lines shown here yield only limited and incomplete information about the magnetic topological structure. One coordinate axis contains a pair of convergent poles, a second one contains a pair of divergent poles, and the third axis coincides with the neutral line. The field configuration is thus essentially three-dimensional, and twodimensional cuts cannot fully represent the global geometry. Hence we concentrate on selected aspects associated with the occurrence of a neutral line inside the magnetosphere, a 

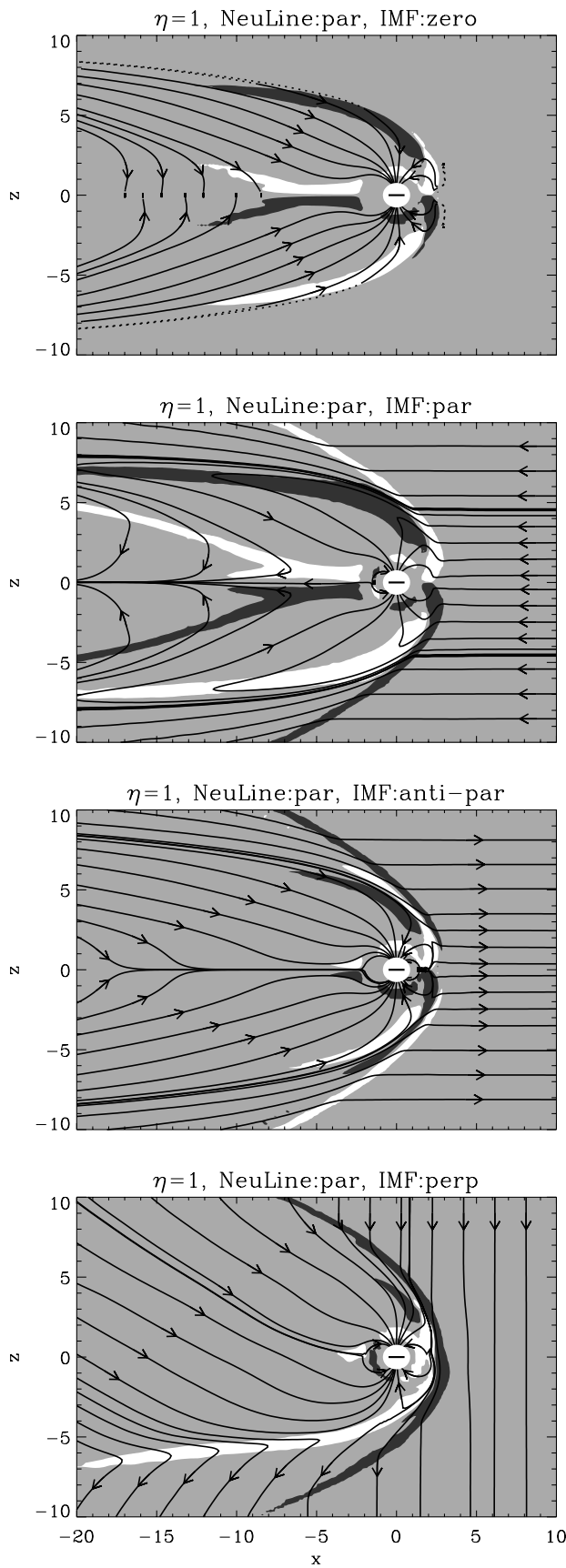

$\eta=1$, NeuLine:perp, IMF:zero

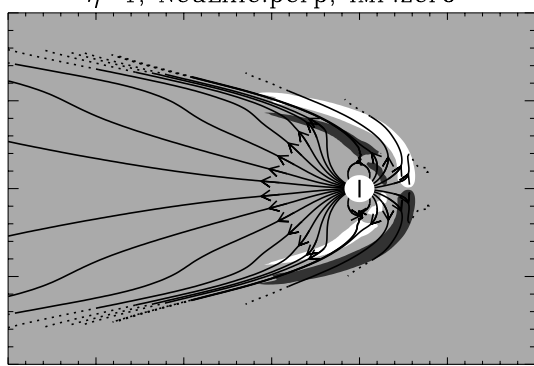

$\eta=1$, NeuLine:perp, IMF:par

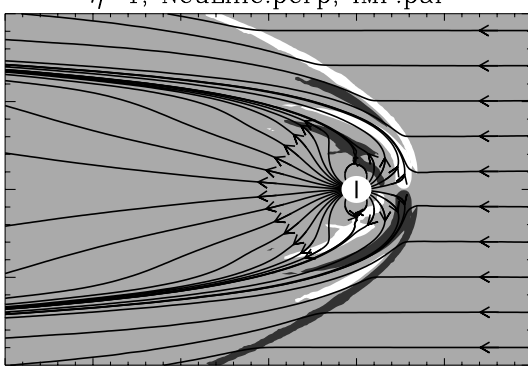

$\eta=1$, NeuLine:perp, IMF:anti-par

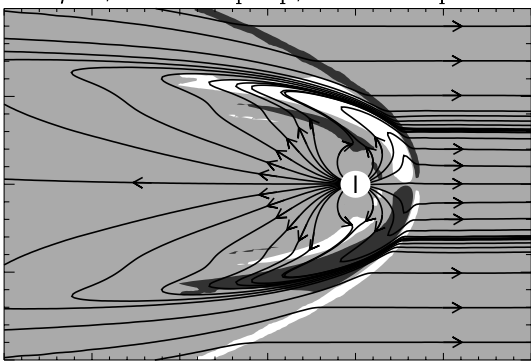

$\eta=1$, NeuLine:perp, IMF:perp

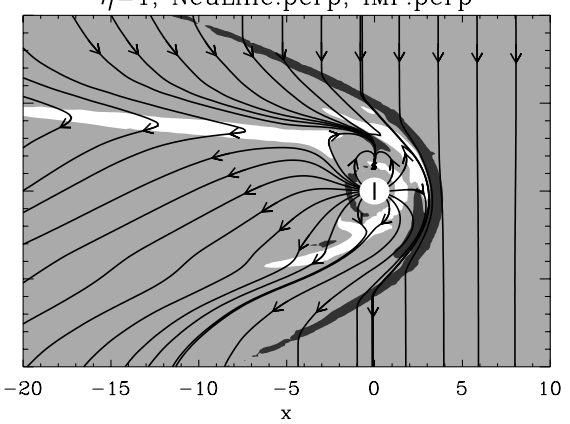

Figure 5. Neutral line quadrupoles as core fields: magnetic field lines (solid) and $y$ component of current density (filled contours) in the plane $y=0$. See color version of this figure at back of this issue.

feature not known from the dipolar or the axisymmetric quadrupolar case.

[41] Modeling the interaction of an $\eta=1$ quadrupole with a zero IMF solar wind turned out to be particularly challenging not only because the step-like increase of magnetic field intensity at the magnetopause must be properly resolved. Small residual field strengths in supposedly zero field regions without any dynamical effect can easily lead to incorrect directions and thus affect the result of field line tracing techniques (note that field lines are usually constructed on the basis of the normalized field). In order to indicate the regions where field line tracing is subject to these errors, field lines in the upper two panels of Figure 5 are dotted in those regions where the field strength is smaller than some threshold (here chosen to be $1 \mathrm{nT}$ ) and are finally cut when the field strength becomes marginal (smaller than $0.1 \mathrm{nT}$ ).

\subsection{Neutral Axis Parallel to the Solar Wind Flow}

[42] We first consider the geometry where one would anticipate a large solar wind impact onto the magnetospheric configuration, namely, the case where the weak field region associated with the neutral line is directly exposed to the approaching solar wind.

[43] For zero IMF conditions (upper left panel in Figure 5) the existence of a neutral line is apparent from the sudden 
termination of otherwise well-behaved field lines in the tail and at the dayside magnetopause. However, it should be noted that this happens only in special planes like the one displayed and the one perpendicular to it which also contains the neutral line. Field lines which are not exactly in such a plane simply avoid the zero field region. If compared with the axisymmetric quadrupole case, the dayside magnetopause stand-off distance is only very little affected by the presence of a weak field region because magnetic flux seems to come in from the flanks. Five singular points can be identified on the dayside magnetopause: the one at the nose originates from the neutral line, and the other four are cusp-like structures associated with the four poles. Only two are visible in the figure plane, the other two are located in the plane perpendicular to it. As can be inferred from symmetry arguments, ChapmanFerraro currents form vortices around the four cusps. Current flow in the tail region is shown in the upper left panel of Figure 6. Magnetopause currents close through an $x$-shaped system of cross-tail currents with the neutral line in its center which leads to an $\otimes$-shaped tail current system.

[44] We now turn to the antiparallel IMF case. There are two planes which contain the IMF direction and one of the poles, and the first one of these $(y=0)$ is shown in the third panel on the left-hand side of Figure 5. If compared with the zero IMF case, the singular point at the nose of the magnetopause has moved further inward, and the null field region in the tail has vanished. Interplanetary magnetic field lines connect to the inner magnetosphere only very little on the dayside and are parallel to the magnetopause field at the flanks of the magnetosphere. The resulting reduction in magnetic shear at the tail magnetopause leads to a decrease in intensity of the tail current system consisting of magnetopause currents and the $\times$-shaped cross-tail currents, see also the third panel on the left-hand side of Figure 6. The structure of the tail current system remains basically unchanged. The bow shock current and the magnetosheath current have no connection to the magnetosphere. To summarize, judging from the third panels on the left-hand sides of Figures 5 and 6, the configuration looks very much like a closed-type magnetosphere.

[45] This interpretation is changed if we look at the lower panel of Figure 7. The diagram shows the configuration in the plane $z=0$ (perpendicular to the first one). The pole here is divergent instead of convergent, which means that the emerging field lines have opposite orientation with respect to the IMF. The resulting configuration differs considerably. Instead of a singular point inside the dayside magnetopause we now find such a point in the tail region. Interplanetary magnetic field lines are connected with the terrestrial field over the whole magnetospheric cross-section line. Magnetic flux penetrates through the flanks of the magnetopause where the interplanetary field lines now find antiparallel magnetospheric counterparts. Compared with the zero IMF case, the magnetopause currents are more intense and so are the associated cross-tail currents.

[46] The configuration for parallel IMF is topologically identical with the antiparallel IMF case but rotated by $90^{\circ}$ around the Sun-Earth line, see the second panels on the lefthand sides of Figures 5 and 6 as well as the upper panel of Figure 7.
[47] The lower left panel of Figure 5 displays the magnetospheric configuration for perpendicular IMF conditions. In the lower hemisphere the field geometry supports cusp reconnection which results in enhanced Chapman-Ferraro currents. The weak field region on the dayside is shielded. The tail current system shown in the lower left panel of Figure 6 does no longer exhibit a clear $\otimes$-shaped geometry: only the upper two legs seem to be intact, the lower two legs and magnetopause currents now follow three-dimensional flow paths.

\subsection{Neutral Axis Perpendicular to the Solar Wind Flow}

[48] We finally look at the case of a quadrupole with a neutral line oriented perpendicular to the solar wind flow direction. The panels on the right-hand sides of Figures 5 and 6 show the magnetospheric field topology and the tail currents for the IMF conditions: zero, parallel, antiparallel, and perpendicular. In all cases the neutral line terminates at two singular points in the upper and lower hemisphere halfway between the Earth's surface and the magnetopause. Whether or not the solar wind has access to a singular point depends on the IMF condition. The parallel IMF case (second right panel of Figure 5) does not exhibit signatures of field line merging, at least not in the plane which contains the neutral line shown here. For the antiparallel IMF case (third panel on the right-hand side of the same figure), on the other hand, basically all planetary field lines in the plane $y=0$ connect to the IMF. The perpendicular IMF case (lower right panel) indicates that reconnection occurs at the dayside and in the magnetotail.

[49] The zero IMF case shown in the upper right panel of Figure 5 seems to indicate that four current systems are present in this plane. The outermost current system must be the Chapman-Ferraro current on the magnetopause which should coincide with the outermost magnetic field line, however, field line tracing in this region is hampered by small field values as explained above. However, the upper right panel of Figure 6 reveals that the dominant part of the tail current system is flowing outside the $y=0$ plane in the form of two banana-shaped closed current systems on either side of that plane. Each current system consists of a cross-tail part and a magnetopause part. The second and third panels of Figure 6 show that the tail current system remains structurally intact if parallel or antiparallel IMF components are present. As for the parallel neutral line orientation and also for the axisymmetric quadrupole case, bow shock currents and magnetosheath currents are flowing around the magnetosphere without connecting to the inner part. The tail current system becomes significantly distorted if perpendicular IMF components are present, the two banana-shaped current systems are still partially intact but now connect to each other and are essentially three-dimensional.

\section{Conclusions}

[50] This study is supposed to provide a first classification of large-scale current systems and magnetic field topology of quadrupolar magnetospheres. We considered the influence of three factors, namely, the shape parameter of the internal quadrupole field, its orientation relative to the 

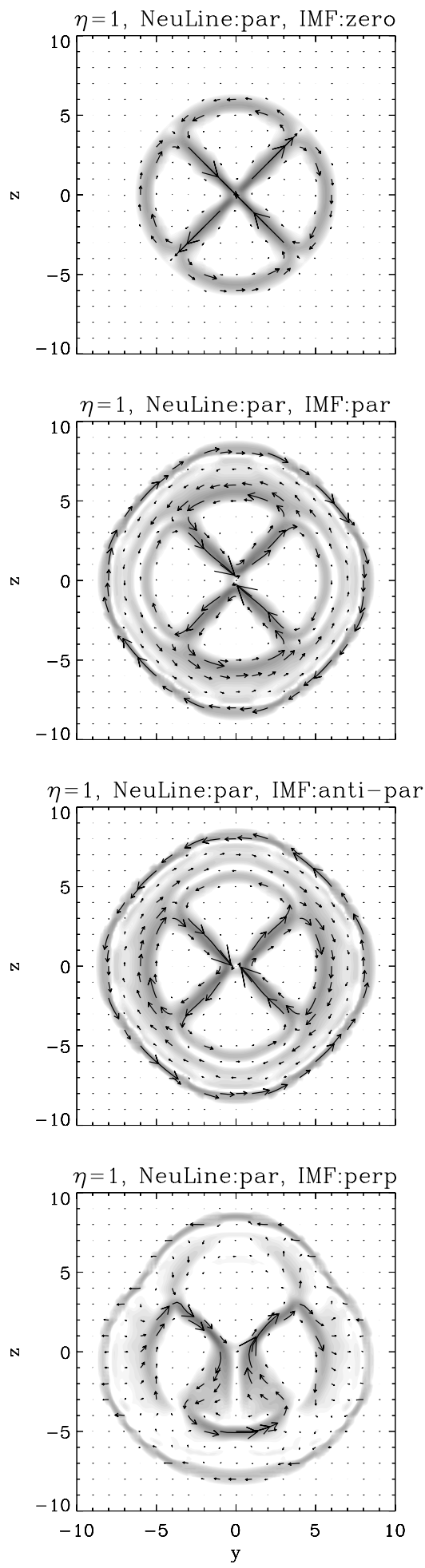
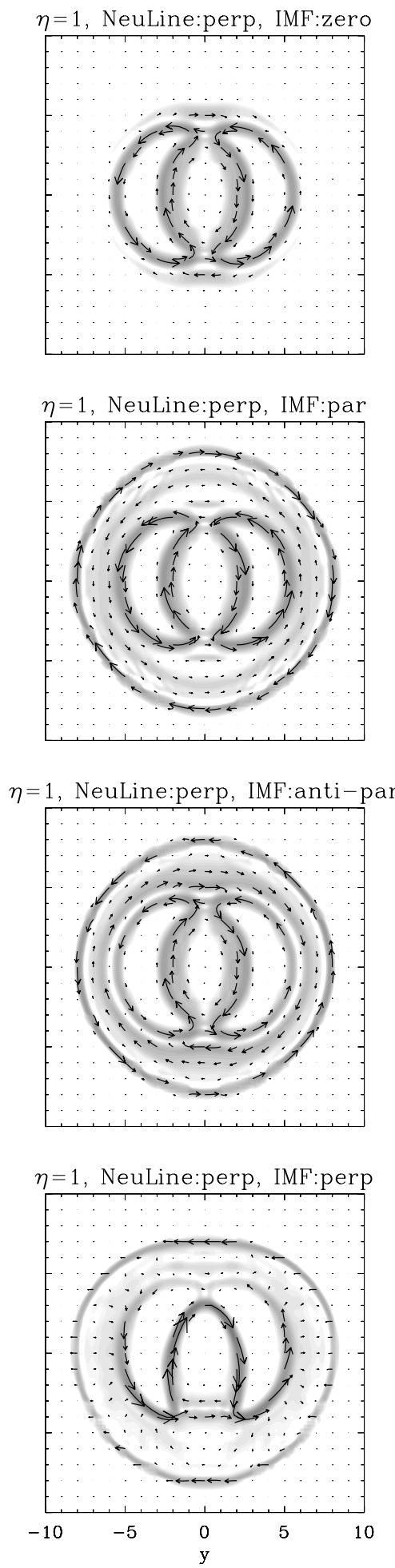

Figure 6. Neutral line quadrupoles as core fields: current vectors projected onto the plane $x=-4 R_{\mathrm{E}}$. See color version of this figure at back of this issue.

solar wind flow direction, and the orientation of the IMF. Owing to the limited scope of this paper, for each factor only very few values could be chosen. Thus we did not yield a complete classification; nonetheless, this study allows to identify some principle structural features in quadrupolar magnetospheres. The results show that all factors have considerable influence on the global magnetospheric configuration.

[51] Of the two topologically different quadrupole fields, the axisymmetric $(\eta=0)$ case yields features which are in many ways similar or at least analogous to dipolar magnetospheres. In both hemispheres of the $\eta=0$ equator-on case 


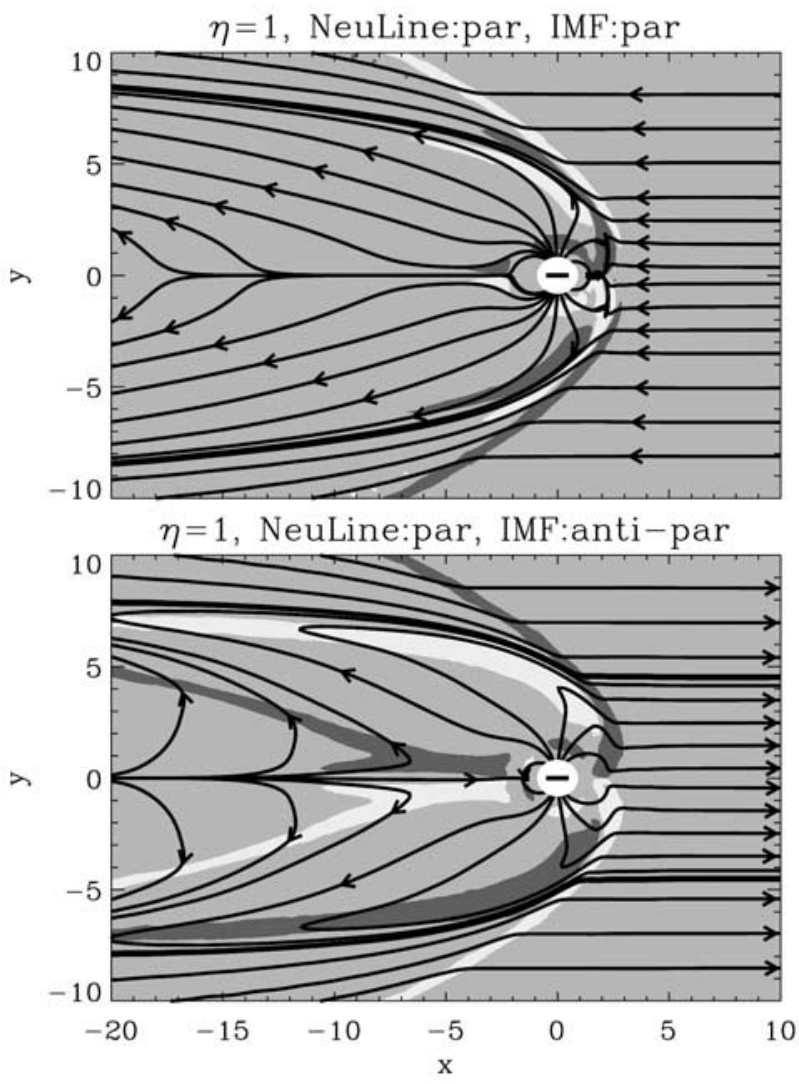

Figure 7. Neutral line quadrupoles as core fields: magnetic field lines (solid) and $z$ component of current density (filled contours) in the plane $z=0$. See color version of this figure at back of this issue.

(where the quadrupole symmetry axis is perpendicular to the solar wind flow) current flow is very similar to the present-day dipolar configuration: one finds a dayside magnetopause current vortex around the cusp combined with a tail current system consisting of a magnetopause current and a cross-tail current. The $\eta=0$ pole-on case yields two circular tail current systems in addition to the magnetopause current, this compares nicely with the circular tail current identified by [Zieger et al., 2004] in simulations of pole-on dipolar magnetospheres. If compared with the results of zero IMF reference cases, purely parallel or antiparallel IMF components do not affect much the field line geometry and the large-scale current systems. In particular, the bow shock current and the magnetosheath current (the latter appears to be pronounced only if there is no perpendicular IMF component) simply flow around the magnetosphere and do not interact with it. Nonzero perpendicular IMF components affect the magnetospheric configuration much stronger. In both the pole-on and the equator-on case there is always one hemisphere at the dayside where the field geometry supports reconnection at the dayside. This yields large-scale transport of plasma and magnetic flux over one of the poles and subsequent tail reconnection, analogous to the classical dipolar field line merging scenario. However, in a quadrupolar magnetosphere only one hemisphere is affected which suggests that solar wind-magnetosphere coupling through reconnection, albeit more or less persistent, should happen on a smaller scale.

[52] Although field configurations are essentially threedimensional, and many aspects do not compare well with the dipolar case, similar conclusions can be drawn for $\eta=1$ magnetospheres. Tail magnetopause currents close through more complex cross-tail currents. Purely parallel or antiparallel IMF components do not change the magnetospheric configuration as much as perpendicular IMF components do. In general, weak field regions that originate from a quadrupolar core field become shielded in the process of magnetosphere formation. This suggests that the influx of high-energy particles into such regions should be less pronounced than expected from the geometry of the the core field. As in the axisymmetric quadrupole case, for any perpendicular IMF orientation the interplanetary magnetic field lines find antiparallel counterparts of planetary origin on the dayside of $\eta=1$ quadrupoles. As a result, field line merging should also occur very frequently but on possibly smaller scales. Paleomagnetospheric dynamics should thus be persistent rather than strongly dependent on the IMF orientation and intermittent like in dipolar magnetospheres.

[53] Studies of paleomagnetospheric processes do not only contribute to fundamental magnetospheric science. Since the magnetosphere acts as a filter for high energetic particles of solar or cosmic origin, geomagnetic polarity transitions are supposed to strongly affect fluxes of such particles into the upper atmosphere. Solar protons may cause ozone depletion events [Stephenson and Scourfield, 1992]. Possible associations of high energetic particles with cloud coverage and relationships between solar activity and climate have been suggested [Svensmark and Friis-Christensen, 1997]. Potential biological implications of magnetic reversals have been discussed by Biernat et al. [2002].

[54] This project is part of a multi-institutional modeling effort coordinated within the DFG Priority Program "Geomagnetic Variations." MHD simulations help to identify paleomagnetospheric configurations which are expected to yield significant particle fluxes into the polar regions. A particle tracing scheme developed at the TU Braunschweig [Stadelmann, 2004] is utilized to calculate these fluxes, and then ionization profiles in the upper and middle atmosphere are computed at the University Osnabrück [Quack et al., 2001]. These profiles are important input variables for an atmospheric chemistry model implemented at the University Bremen [Sinnhuber et al., 2003] which computes the resulting ozone concentrations in the middle atmosphere.

\section{Appendix A: Quadrupole Shape Parameter $\eta$}

[55] Any quadrupole magnetic field can be written as $\overrightarrow{\boldsymbol{B}}=$ $-\nabla \Psi$ with the potential

$$
\Psi=\frac{1}{2} \sum_{i, j=1}^{3} Q_{i j} \frac{x_{i} x_{j}}{r^{5}}=\frac{1}{2} \frac{\mathbf{x}^{\dagger} \mathcal{Q} \mathbf{x}}{r^{5}}
$$

[Jackson, 1963], where $r=|\mathbf{x}|$. A quadrupole tensor $\mathcal{Q}$ is symmetric and its trace is zero which implies that a quadrupole field has five degrees of freedom. Following Vogt and Glassmeier [2000], we may apply a convenient rotation of the coordinate system to diagonalize the quadrupole tensor and thereby eliminating three degrees 
of freedom. If we choose the $x_{3}$ axis to be an eigenvector associated with the eigenvalue $q$ of largest absolute value, the quadrupole tensor may be written as

$$
q=\amalg \cdot\left(\begin{array}{ccc}
-(1-\eta) / 2 & 0 & 0 \\
0 & -(1+\eta) / 2 & 0 \\
0 & 0 & 1,
\end{array}\right)
$$

which defines the so-called quadrupole shape parameter $\eta \in[-1,1]$. The parameters $q$ and $\eta$ account for the remaining two degrees of freedom. The field topology is governed by $\eta$, and the field strength for a given topology is controlled by $q$.

\section{Appendix B: MHD Simulation Code BATS-R-US}

[56] This section outlines the basic features of the BATSR-US MHD simulation code. For details, the reader is referred to the comprehensive description given by Powell et al. [1999].

[57] The BATS-R-US code solves the equations of ideal magnetohydrodynamics (MHD). Pressure is assumed to be a scalar, the heat flow is zero, and the conductivity is taken to be infinite. The so-called conservative form of this set of equations is

$$
\begin{gathered}
\frac{\partial \rho}{\partial t}+\nabla \cdot(\rho \mathbf{u})=0, \\
\frac{\partial(\rho \mathbf{u})}{\partial t}+\nabla \cdot\left[\rho \mathbf{u} \mathbf{u}+\left(p+\frac{B^{2}}{2 \mu_{0}}\right) \mathcal{I}-\frac{\mathbf{B} \mathbf{B}}{\mu_{\prime}}\right]=0, \\
\frac{\partial \mathbf{B}}{\partial t}+\nabla \cdot(\mathbf{u} \mathbf{B}-\mathbf{B} \mathbf{u})=0, \\
\frac{\partial \varepsilon}{\partial t}+\nabla \cdot\left[\mathbf{u}\left(\varepsilon+p+\frac{B^{2}}{2 \mu_{0}}\right)-\frac{(\mathbf{u} \cdot \mathbf{B}) \mathbf{B}}{\mu_{0}}\right]=0 .
\end{gathered}
$$

Here $\rho$ is the plasma mass density, $\mathbf{u}$ is the plasma velocity, $\mathbf{B}$ is the magnetic field, $p$ is the pressure, and $\varepsilon$ is the total energy density given by

$$
\varepsilon=\frac{\rho u^{2}}{2}+\frac{p}{\gamma-1}+\frac{B^{2}}{2 \mu_{0}} .
$$

Note that the Earth's gravity was neglected in the simulations described here. The boundary conditions were chosen to be fixed solarwind values at the inflow (maximum $x=$ antisunward) boundary, zero extrapolation at the outflow (minimum $x=$ sunward) boundary, and zero gradient (Neumann) conditions at the $y$ and $z$ boundaries. At the inner boundary representing the interface to the planet Earth, the velocity and the radial component of the deviation magnetic field were fixed to be zero, zero gradients were assumed for the tangential components of the magnetic field, and the mass density and the pressure were fixed. Although this combination of settings cannot

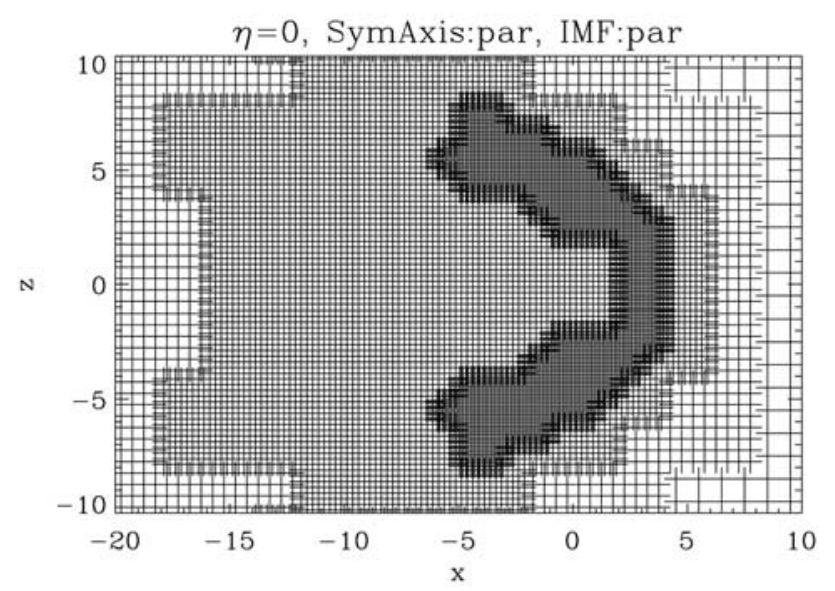

Figure B1. Example of a numerical grid resulting from adaptive mesh refinement in the MHD simulation code BATS-R-US.

represent the complexity of ionospheric physics, simulation runs of dipolar magnetospheres have proven to mimic the response of a highly conductive ionosphere and to reproduce basic features of equatorial convection.

[58] The BATS-R-US simulation code solves the set of ideal MHD equations in dimensionless form using approximate Riemann solvers on adaptive grids. Different types of Riemann solvers are implemented (Roe, Linde, Rusanov). Adaptive mesh refinement (AMR) allows to make efficient use of computational resources: physically interesting regions (discontinuities, current sheets, steep gradients) can be resolved in sufficient detail without necessarily increasing the grid resolution in other parts of the simulation domain.

[59] In the paleomagnetosphere simulations described in this paper, current sheets were the key regions of interest, so refinement was based on the quantity $\nabla \times \overrightarrow{\boldsymbol{B}}$. The simulation domain $\left[-32 R_{\mathrm{E}}, 32 R_{\mathrm{E}}\right]^{3}$ was divided into about 35,000 blocks of $4^{3}$ cells each, yielding a total number of more than 2 million cells. The size of the cells varied from $0.125 R_{\mathrm{E}}$ to $8 R_{\mathrm{E}}$. Figure $\mathrm{B} 1$ shows the grid structure for one of the simulation runs.

[60] Acknowledgments. The German Research Council DFG provided financial support for JV, BZ, AS, and KHG through grants VO-855/2 and GL-142/12. Simulation results were obtained using BATS-R-US, developed by the Center for Space Environment Modeling at the University of Michigan with funding support from NASA ESS, NASA ESTO-CT, NSF KDI, and DoD MURI. Simulation runs were carried out on highperformance computing facilities of the CLAMV at International University Bremen, of the Research Center Juelich, and of the TU Braunschweig.

[61] Lou-Chuang Lee thanks Helfried Biernat and Gerd-Hannes Voigt for their assistance in evaluating this paper.

\section{References}

Baumjohann, W., and R. A. Treumann (1997), Basic Space Plasma Physics, Imperial Coll. Press, London.

Biernat, H. K., N. I. Kömle, and H. I. M. Lichtenberger (1985), Analytical two-dimensional model of a quadrupole magnetosphere, Planet. Space Sci., 33, 45-52

Biernat, H. K., N. V. Erkaev, T. Penz, H. Lammer, S. C. Manrubia, F. Selsis, D. F. Vogl, and S. Mühlbacher (2002), Magnetic field reversals on earth: Possible implications for the biosphere, in Proceedings of the Second European Workshop on Exo/Astrobiology, ESA SP-518, Eur. Space Agency, Paris. 
Clement, B. M. (1991), Geographical distribution of transitional VGP's: Evidence for non-zonal equatorial symmetry during the MatuyamaBrunhes geomagnetic reversal, Earth Planet. Sci. Lett., 104, 48-58.

Clement, B. M., and D. V. Kent (1985), A comparison of two sequential polarity transitions (upper Olivai and lower Jaramillo) from the southern hemisphere, Phys. Earth Planet. Int., 39, 310-313.

Dungey, J. W. (1961), Interplanetary magnetic field and the auroral zones, Phys. Rev. Lett., 6, 47-48.

Erickson, G. A., and R. A. Wolf (1980), Is steady state convection possible in the earth's magnetotail?, Geophys. Res. Lett., 7, 897-900.

Fedder, J. A., S. P. Slinker, and J. G. Lyon (1998), A comparison of global numerical simulation results to data for the January 27-28, 1992, Geospace Environment Modeling challange event, J. Geophys. Res., 103, $14,799-14,810$

Glatzmaier, G. A., and P. H. Roberts (1996), Rotation and magnetism of Earth's inner core, Science, 274, 1887-1891.

Gombosi, T. I., D. L. DeZeeuw, C. P. T. Groth, K. G. Powell, and P. Song (1998), The length of the magnetotail for northward IMF: Results of 3D MHD simulations, in Physics of Space Plasmas, vol. 15, edited by T. Chang and J. R. Jasperse, pp. 121-128, Cent. for Theor. Geo/Cosmo Plasma Phys., Mass. Inst. of Technol., Cambridge, Mass.

Haerendel, G. (2001), Reconnection, in The Century of Space Science, pp. 1007-1033, Springer, New York.

Jackson, J. D. (1963), Classical Electrodynamics, John Wiley, Hoboken, N. J.

Jacobs, J. A. (1994), Reversals of the Earth's Magnetic Field, Cambridge Univ. Press, New York.

Janhunen, P., T. I. Pulkkinen, and K. Kauristie (1995), Auroral fading in ionosphere-magnetosphere coupling model: Implications for possible mechanisms, Geophys. Res. Lett., 22, 2049-2052.

Kabin, K., R. Rankin, R. Marchand, T. I. Gombosi, C. R. Clauer, A. J. Ridley, V. O. Papitashvili, and D. L. DeZeeuw (2003), Dynamic response of the Earth's magnetosphere to By reversals, J. Geophys. Res., 108(A3), 1132, doi:10.1029/2002JA009480.

Leubner, M. P., and K. Zollner (1985), The quadrupole magnetopause, J. Geophys. Res., 90, 8265-8268.

Lyon, J. G., R. E. Lopez, C. C. Goodrich, M. Wiltberger, and K. Papadopoulos (1998), Simulation of the March 9, 1995, substorm Auroral brightening and the onset of lobe reconnection, Geophys. Res. Lett., 25, 3039-3042.

Martyn, D. F. (1951), The theory of magnetic storms and auroras, Nature $167,92-94$

Merrill, R. T., and M. W. McElhinny (1983), The Earth's Magnetic Field, Int. Geophys. Ser, vol. 32, Elsevier, New York.

Merrill, R. T., and P. L. McFadden (1999), Geomagnetic polarity transitions, Rev. Geophys., 37, 201-226.

Nakata, H., M. Shinohara, Y. Kamide, and T. Ogino (2004), Ionospheric electric potentials for substorms calculated from a solar wind-magnetosphere MHD simulation and a magnetogram inversion technique, J. Geophys. Res., 109, A01211, doi:10.1029/2002JA009743.

Ohtani, S., and J. Raeder (2004), Tail current surge: New insights from a global MHD simulation and comparison with satellite observations, J. Geophys. Res., 109, A01207, doi:10.1029/2002JA009750.

Powell, K. G., P. L. Roe, T. J. Linde, T. I. Gombosi, and D. L. DeZeeuw (1999), A solution-adaptive upwind scheme for ideal magnetohydrodynamics, J. Comput. Phys., 154, 284-309.

Priest, E., and T. Forbes (2000), Magnetic Reconnection: MHD Theory and Applications, Cambridge Univ. Press, New York.

Quack, M., M.-B. Kallenrode, M. von König, K. Künzi, J. Burrows, B. Heber, and E. Wolff (2001), Ground level events and consequences for stratospheric chemistry, in Proceedings of ICRC 2001, Copernicus Gesellschaft, Katlenburg-Lindau, Germany.

Rae, I. J., K. Kabin, R. Rankin, F. R. Fenrich, W. Liu, J. A. Wanliss, A. J. Ridley, T. I. Gombosi, and D. L. DeZeeuw (2004), Comparison of photometer and global MHD determination of the open-closed field line boundary, J. Geophys. Res., 109, A01204, doi:10.1029/2003JA009968.

Raeder, J., J. Berchem, and M. Ashour-Abdalla (1998), The Geospace Environment Modeling Grand Challenge: Results from a global geospace circulation model, J. Geophys. Res., 103, 14,787-14,798.

Raeder, J., R. L. McPherron, L. A. Frank, S. Kokubun, G. Lu, T. Mukai, W. R. Paterson, J. B. Sigwarth, H. J. Singer, and J. A. Slavin (2001),
Global simulation of the Geospace Environment Modeling substorm challenge event, J. Geophys. Res., 106, 381-395.

Rishbeth, H. (1985), The quadrupole ionosphere, Ann. Geophys., 3, $293-$ 298.

Saito, T., T. Sakurai, and K. Yumoto (1978), The Earth's palaeomagnetosphere as the third type of planetary magnetosphere, Planet. Space Sci., $26,413-422$

Sinnhuber, M., J. P. Burrows, M. P. Chipperfield, C. H. Jackman, M.-B. Kallenrode, K. F. Künzi, and M. Quack (2003), A model study of the impact of magnetic field structure on atmospheric composition during solar proton events, Geophys. Res. Lett., 30(15), 1818, doi:10.1029/ 2003GL017265.

Siscoe, G. L. (1979), Towards a comparative theory of magnetospheres, in Solar System Plasma Physics, vol. II, pp. 319-402, Elsevier, New York. Siscoe, G. L., and C.-K. Chen (1975), The paleomagnetosphere, J. Geophys. Res., 80, 4675-4680.

Siscoe, G. L., and N. J. Crooker (1976), Auroral zones in a quadrupole magnetosphere, J. Geomagn. Geoelectr., 28, 1-9.

Siscoe, G. L., G. M. Erickson, B. U. Ö. Sonnerup, N. C. Maynard, J. A. Schoendorf, K. D. Siebert, D. R. Weimer, W. W. White, and G. R. Wilson (2002), Hill model of transpolar potential saturation: Comparisons with MHD simulations, J. Geophys. Res., 107(A6), 1075, doi:10.1029/ 2001JA000109

Slinker, S. P., J. A. Fedder, J. M. Ruohoniemi, and J. G. Lyon (2001), Global MHD simulation of the magnetosphere for November 24, 1996 , J. Geophys. Res., 106, 361-380.

Stadelmann, A. (2004), Globale Effekte einer Erdmagnetfeldumkehr: Magnetosphärenstruktur und kosmische Teilchen, dissertation, Tech. Univ., Braunschweig, Germany.

Starchenko, S., and V. Shcherbakov (1991), Inverse magnetosphere (in Russian), Dokl. Akad. Nauk SSSR, 321, 69-74.

Stephenson, J. A. E., and M. W. J. Scourfield (1992), Ozone depletion over the polar caps caused by solar protons, Geophys. Res. Lett., 19, 24252428 .

Svensmark, H., and E. Friis-Christensen (1997), Variation of cosmic ray flux and global cloud coverage-A missing link in solar-climate relationship, J. Atmos. Sol. Terr. Phys., 59, 1225-1232.

Vogt, J., and K.-H. Glassmeier (2000), On the location of trapped particle populations in quadrupole magnetospheres, J. Geophys. Res., 105, $13,063-13,071$

Vogt, J., and K.-H. Glassmeier (2001), Modelling the paleomagnetosphere: Strategy and first results, Adv. Space Res., 28, 863-868.

White, W. W., J. A. Schoendorf, K. D. Siebert, N. C. Maynard, D. R. Weimer, G. R. Wilson, B. U. Ö. Sonnerup, G. L. Siscoe, and G. M. Erickson (2001), MHD simulation of magnetospheric transport at the mesocale, in Space Weather, Geophys. Monogr. Ser., vol. 125, edited by P. Song, H. J. Singer, and G. L. Siscoe, pp. 229-240, AGU, Washington, D. C.

Williams, I., and M. Fuller (1981), Zonal harmonic models of reversal transition fields, J. Geophys. Res., 86, 11,657-11,665.

Willis, D. M., A. C. Holder, and C. J. Dais (2000), Possible configurations of the magnetic field in the outer magnetosphere during geomagnetic polarity reversals, Ann. Geophys., 18, 11-27.

Winglee, R. M., and J. D. Menietti (1998), Auroral activity associated with pressure pulses and substorms: A comparison between global fluid modeling and Viking UV imaging, J. Geophys. Res., 103, 9189-9206.

Zieger, B., J. Vogt, K.-H. Glassmeier, and T. I. Gombosi (2004), Magnetohydrodynamic simulation of an equatorial dipolar paleomagnetosphere, J. Geophys. Res., 109, A07205, doi:10.1029/2004JA010434.

K.-H. Glassmeier and A. Stadelmann, Institut für Geophysik und extraterrestrische Physik, Technische Universität, Braunschweig, Mendelssohnstr. 3, 38106 Braunschweig, Germany.

T. I. Gombosi, K. C. Hansen, and A. J. Ridley, University of Michigan, Ann Arbor, MI 48109-2143, USA.

J. Vogt and B. Zieger, School of Engineering and Science, International University Bremen, P.O. Box 750561, 28725 Bremen, Germany. (j.vogt@iu-bremen.de) 

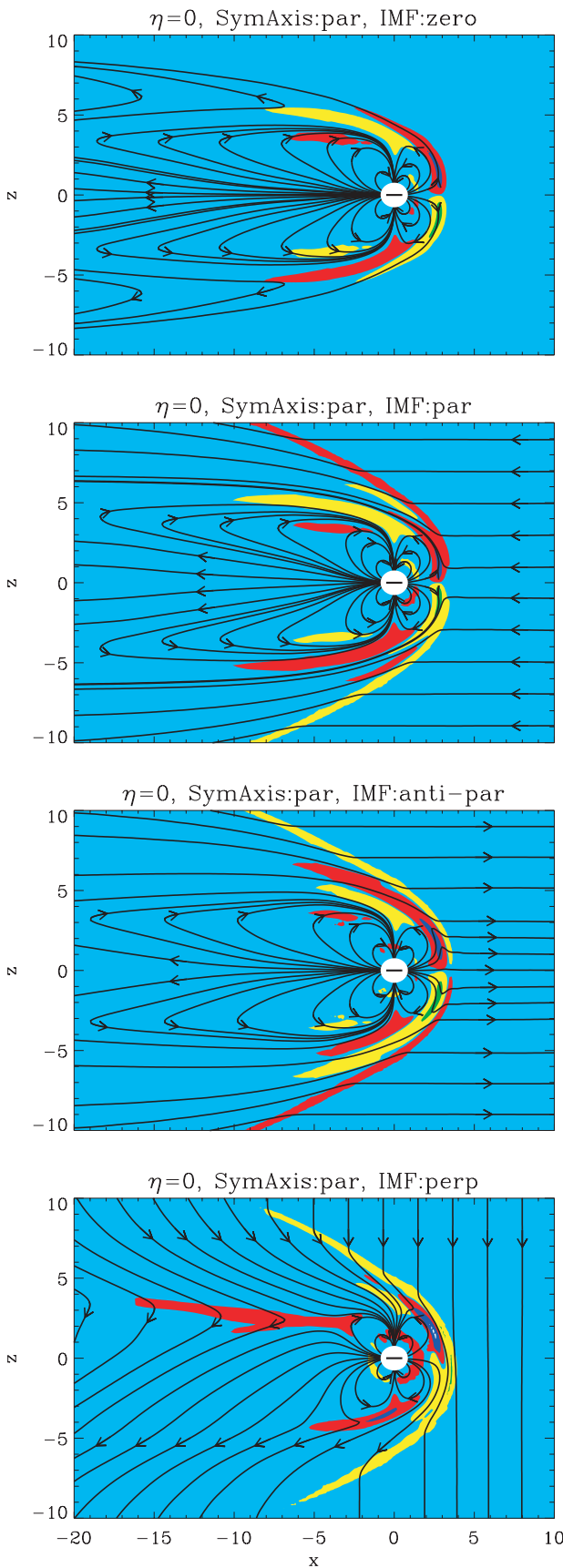

$\eta=0$, SymAxis:perp, IMF:zero

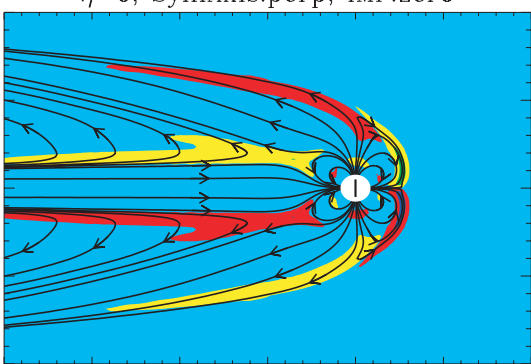

$\eta=0$, SymAxis:perp, IMF:par

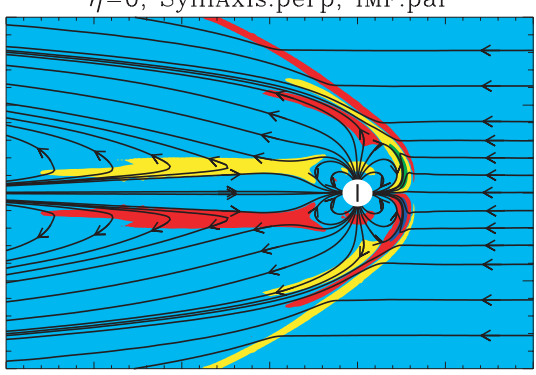

$\eta=0$, SymAxis:perp, IMF:anti-par

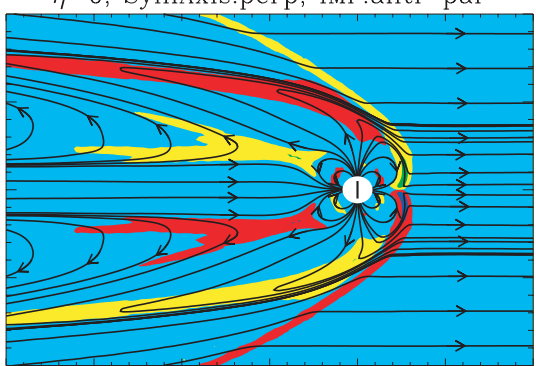

$\eta=0$, SymAxis:perp, IMF:perp

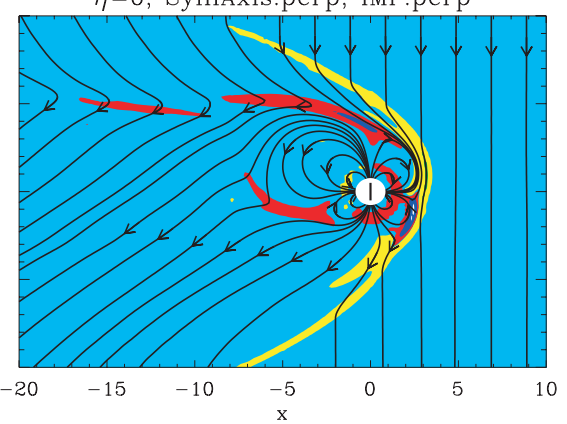

Figure 2. Axisymmetric quadrupolar core fields: magnetic field lines (solid) and $y$ component of current density (filled contours) in the plane $y=0$. 

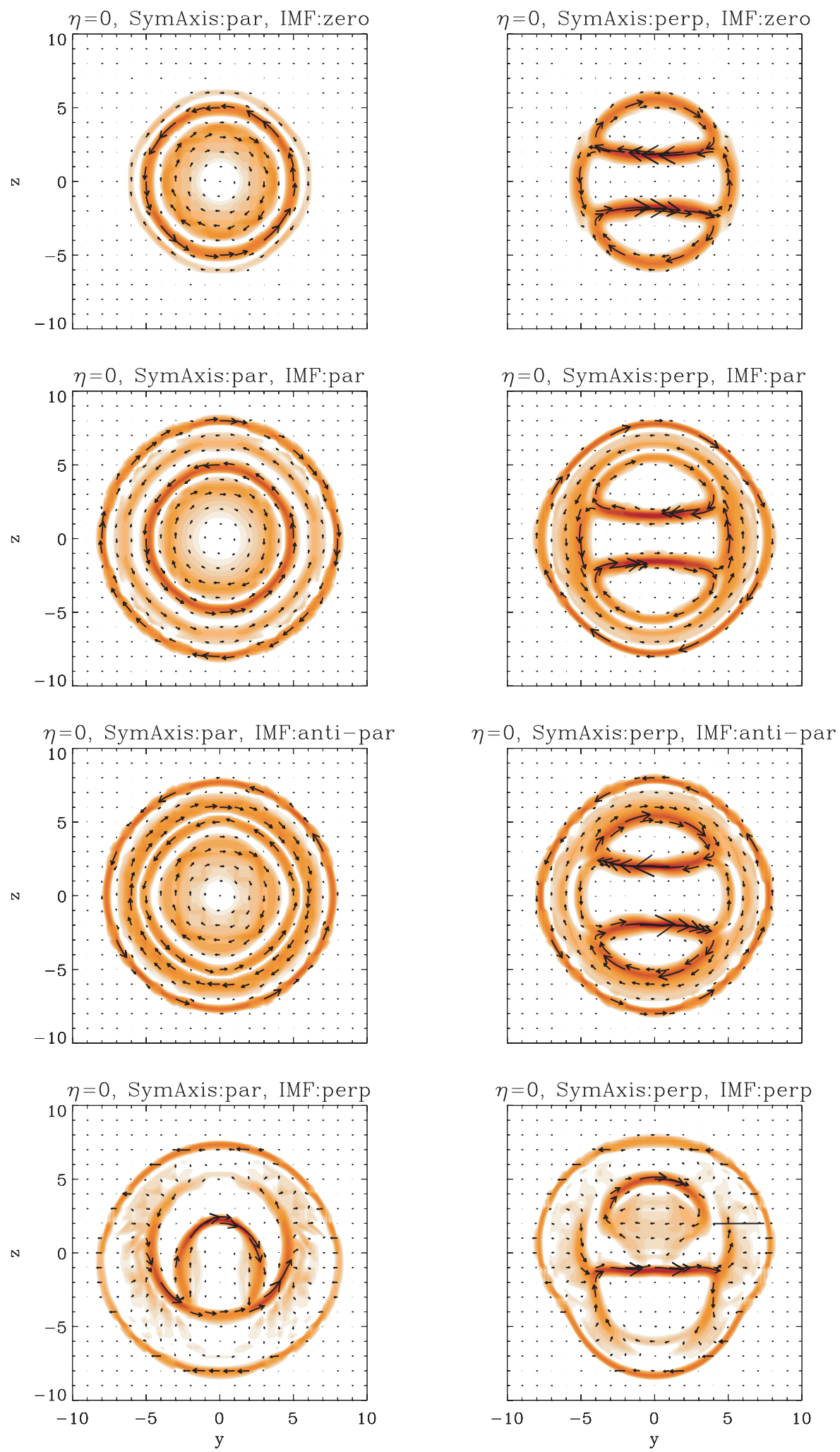

Figure 3. Axisymmetric quadrupolar core fields: current vectors projected onto the plane $x=-4 R_{\mathrm{E}}$. 

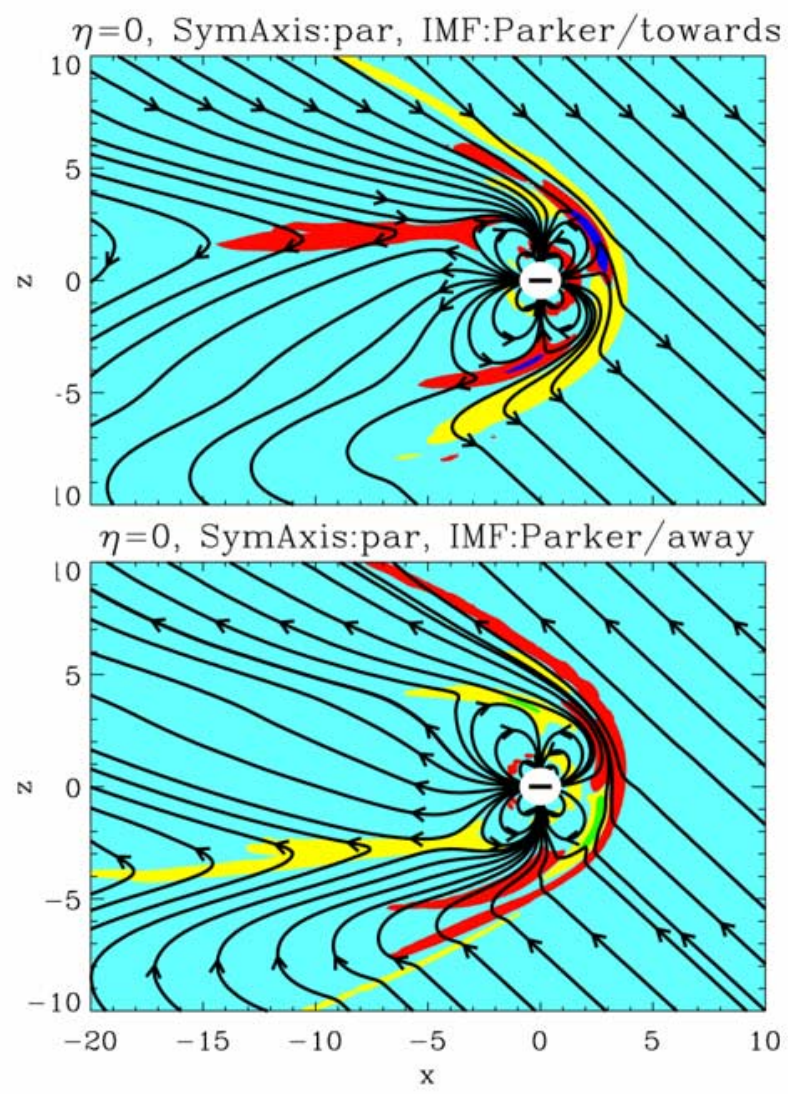

Figure 4. Axisymmetric quadrupolar core fields, oblique IMF: magnetic field lines (solid) and $y$ component of current density (filled contours) in the plane $y=0$. 

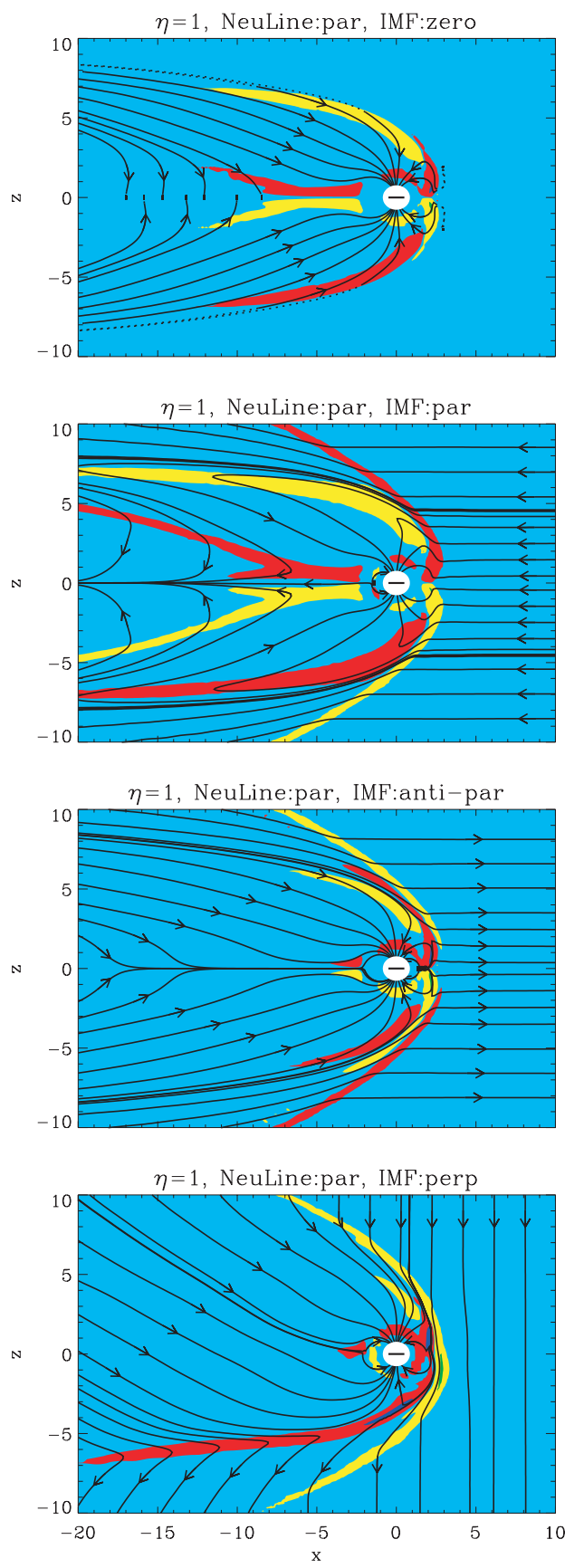

$\eta=1$, NeuLine:perp, IMF:zero

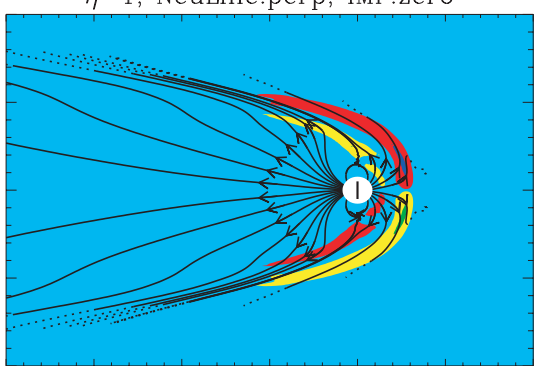

$\eta=1$, NeuLine:perp, IMF:par

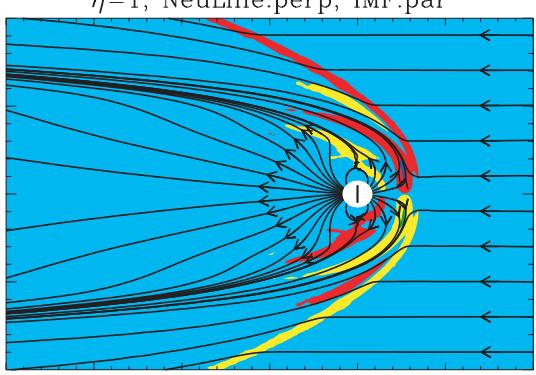

$\eta=1$, NeuLine:perp, IMF:anti-par

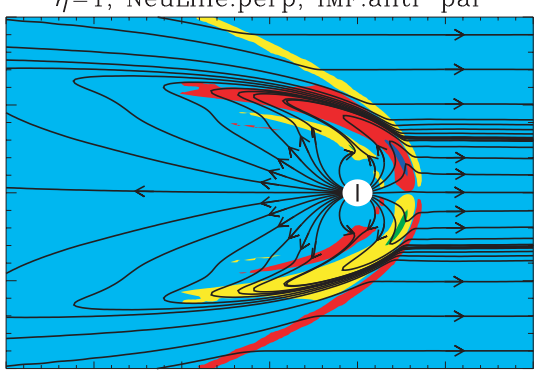

$\eta=1$, NeuLine:perp, IMF:perp

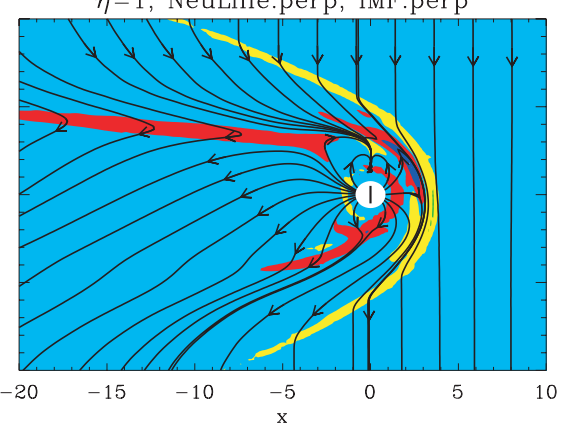

Figure 5. Neutral line quadrupoles as core fields: magnetic field lines (solid) and $y$ component of current density (filled contours) in the plane $y=0$. 

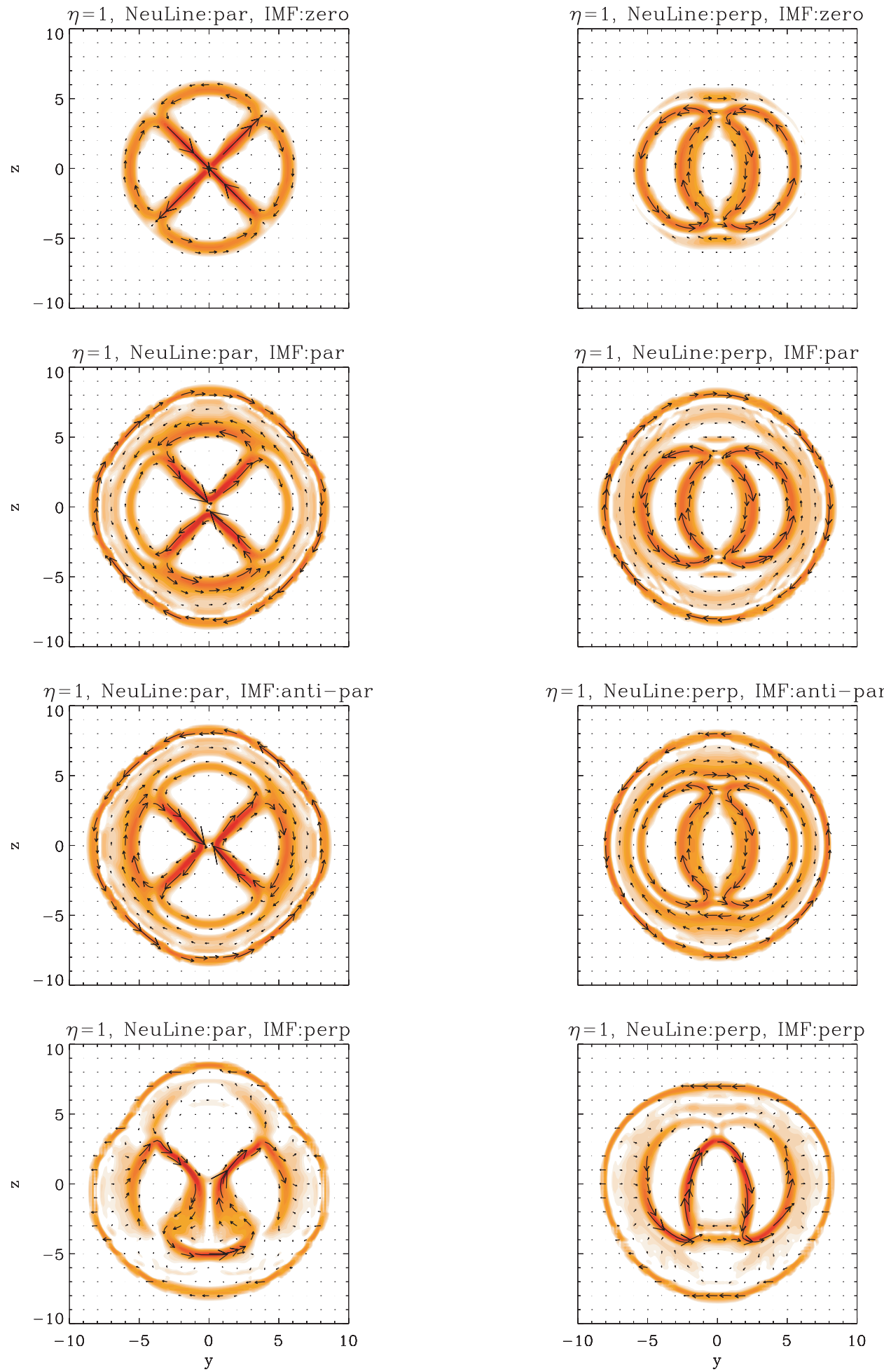

Figure 6. Neutral line quadrupoles as core fields: current vectors projected onto the plane $x=-4 R_{\mathrm{E}}$. 

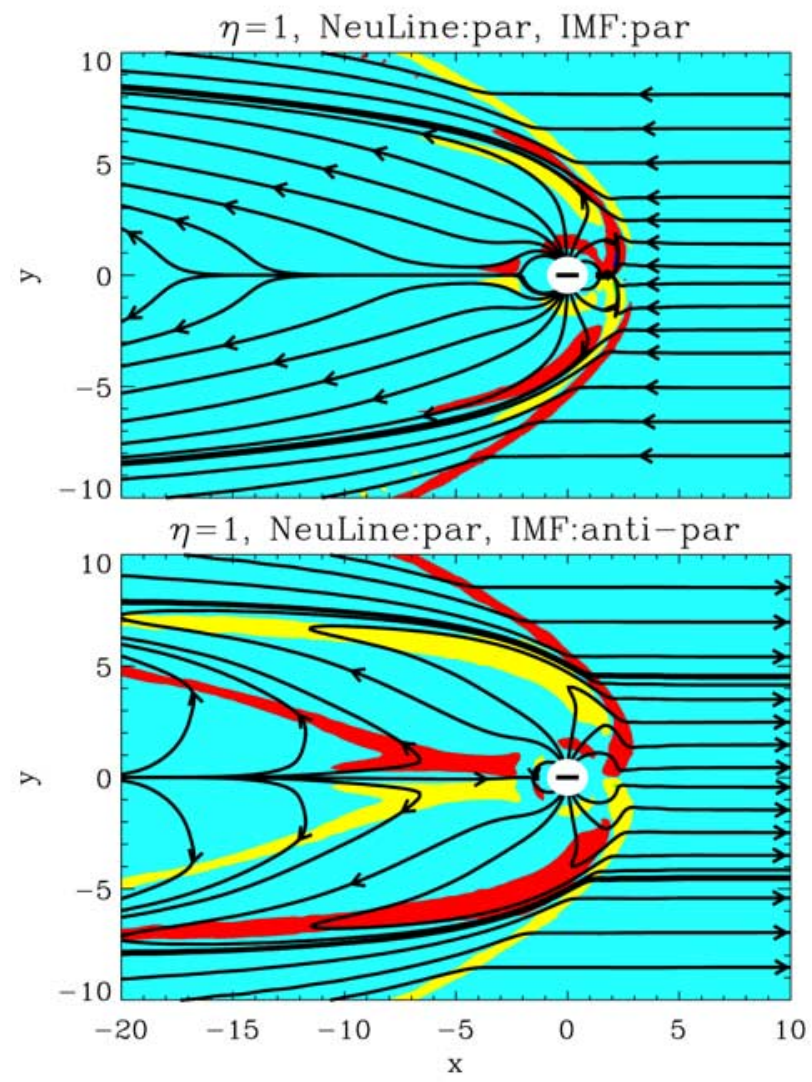

Figure 7. Neutral line quadrupoles as core fields: magnetic field lines (solid) and $z$ component of current density (filled contours) in the plane $z=0$. 